\title{
A VARIANT OF CLARK'S THEOREM AND ITS APPLICATIONS FOR NONSMOOTH FUNCTIONALS WITHOUT THE PALAIS-SMALE CONDITION*
}

\author{
SHAOWEI CHEN ${ }^{\dagger}$, ZHAOLI LIU ${ }^{\ddagger}$, AND ZHI-QIANG WANG§
}

\begin{abstract}
By introducing a new notion of the genus with respect to the weak topology in Banach spaces, we prove a variant of Clark's theorem for nonsmooth functionals without the Palais-Smale condition. In this new theorem, the Palais-Smale condition is replaced by a weaker assumption, and a sequence of critical points converging weakly to zero with nonpositive energy is obtained. As applications, we obtain infinitely many solutions for a quasi-linear elliptic equation which is very degenerate and lacks strict convexity, and we also prove the existence of infinitely many homoclinic orbits for a second-order Hamiltonian system for which the functional is not in $C^{1}$ and does not satisfy the Palais-Smale condition. These solutions cannot be obtained via existing abstract theory.
\end{abstract}

Key words. the Clark theorem, weak genus, very degenerate quasi-linear equation, homoclinic orbit of Hamiltonian system

AMS subject classifications. 35J20, 35J60

DOI. $10.1137 / 15 \mathrm{M} 1034635$

1. Introduction and main result. The Clark theorem (see [6]) is a classical theorem in critical point theory that has numerous applications in the existence theory of differential equations and dynamic systems (see, for instance, $[5,16,20,21,23,26$, 27]). In [16], Heinz stated the Clark theorem in the following form.

Theorem A (see [16]). Let $X$ be a Banach space, $J \in C^{1}(X, \mathbb{R})$. Assume $J$ satisfies the Palais-Smale condition, is even and bounded from below, and $J(0)=0$. If for any $k \in \mathbb{N}$, there exists a $k$-dimensional subspace $X_{k}$ of $X$ and $\rho_{k}>0$ such that $\sup _{X_{k} \cap S_{\rho_{k}}} J<0$, where $S_{\rho}=\{u \in X \mid\|u\|=\rho\}$, then $J$ has a sequence of critical values $c_{k}<0$ satisfying $c_{k} \rightarrow 0$ as $k \rightarrow \infty$.

This theorem was improved by Kajikiya in [18], where it is proved that under the same assumptions as in Theorem A, there exists a sequence $\left\{u_{k}\right\}$ of critical points of $J$ such that $J\left(u_{k}\right) \leq 0, u_{k} \neq 0$, and $\lim _{k \rightarrow \infty}\left\|u_{k}\right\|=0$. The additional information $\lim _{k \rightarrow \infty}\left\|u_{k}\right\|=0$ of the critical point sequence has been proved to be very important in many applications (see [18] and [21]). In [21], Liu and Wang extended the Clark theorem in various situations. As well as giving a new proof for the Kajikiya result, they proved a new version of the Clark theorem for nonsmooth functionals which can

\footnotetext{
${ }^{*}$ Received by the editors August 11, 2015; accepted for publication (in revised form) November 7, 2016; published electronically February 16, 2017.

http://www.siam.org/journals/sima/49-1/M103463.html

Funding: The work of the first author was supported by the Science Foundation of Huaqiao University (13BS208) and the Promotion Program for Young and Middle-aged Teachers in Science and Technology Research of Huaqiao University (ZQN-PY119). The work of the second author was supported by NSFC-11271265, NSFC-11331010, NSFC-11671272, and BCMIIS. The work of the third author was supported by NSFC-11271201.

${ }^{\dagger}$ School of Mathematical Sciences, Huaqiao University, Quanzhou 362021, People's Republic of China (swchen6@163.com).

${ }^{\ddagger}$ School of Mathematical Sciences, Capital Normal University, Beijing 100048, People’s Republic of China (zliu@cnu.edu.cn).

$\S$ Center for Applied Mathematics, Tianjin University, Tianjin 300072, People's Republic of China, and Department of Mathematics and Statistics, Utah State University, Logan, UT 84322 (zhi-qiang. wang@usu.edu).
} 
be applied to various quasi-linear elliptic equations for the existence of infinitely many solutions. They also gave a surprising variant of the Clark theorem for strongly indefinite functionals and used it to obtain infinitely many periodic solutions for sublinear first-order Hamiltonian systems.

However, all those variants of the Clark theorem mentioned above rely on the Palais-Smale condition, which makes it impossible to apply those abstract results to differential equations in many important situations. The present paper is devoted to establishing a new variant of the Clark theorem for nonsmooth functionals which do not satisfy the Palais-Smale condition. The motivations are from applications to very degenerate quasi-linear equations and from applications to homoclinic orbits of Hamiltonian systems. In these situations, we will see that the usual Palais-Smale condition cannot hold and that the sequence of solutions weakly converging to zero cannot converge to zero in the strong norm, let alone in the $L^{\infty}$-norm. The quasilinear elliptic equations considered here are very degenerate and lack strict convexity, and the second-order Hamiltonian systems treated here do not have a $C^{1}$ variational formulation and do not satisfy the Palais-Smale condition.

We recall some notations and definitions before stating our main result. Let $X$ be a Banach space and $X^{\prime}$ be its dual space. The weak convergence in $X$ is denoted by " $\rightarrow$ ". Let $J$ be a functional defined on $X$. $J$ is said to be weakly sequentially lower semicontinuous if $\liminf _{n \rightarrow \infty} J\left(u_{n}\right) \geq J(u)$ for any $u \in X$ and $\left\{u_{n}\right\} \subset X$ satisfying $u_{n} \rightarrow u$.

Let $J$ be a continuous functional defined on $X$ and $E$ a dense subspace of $X$. As in [19], we say that $J$ is $E$-differentiable if

(a) for all $u \in X$ and all $h \in E$, the derivative of $J$ at $u$ in the direction $h$, denoted by $\left\langle J^{\prime}(u), h\right\rangle$, exists, that is,

$$
\left\langle J^{\prime}(u), h\right\rangle=\lim _{t \rightarrow 0+} \frac{J(u+t h)-J(u)}{t} ;
$$

(b) the mapping $(u, h) \mapsto\left\langle J^{\prime}(u), h\right\rangle$ satisfies

(i) $\left\langle J^{\prime}(u), h\right\rangle$ is linear in $h$,

(ii) $\left\langle J^{\prime}(u), h\right\rangle$ is continuous in $u$, that is, $\left\langle J^{\prime}\left(u_{n}\right), h\right\rangle \rightarrow\left\langle J^{\prime}(u), h\right\rangle$ as $u_{n} \rightarrow u$ in $X$.

The slope of an $E$-differentiable functional $J$ at $u$ denoted by $\left|J^{\prime}(u)\right|$ is an extended number in $[0,+\infty]$ :

$$
\left|J^{\prime}(u)\right|=\sup \left\{\left\langle J^{\prime}(u), h\right\rangle \mid h \in E,\|h\|=1\right\},
$$

where $\|\cdot\|$ denotes the norm in $X$. A point $u \in X$ is said to be a critical point of $J$ if $\left|J^{\prime}(u)\right|=0$.

The main result of this paper is the following theorem.

Theorem 1.1. Let $X$ be a separable and reflexive Banach space with norm $\|\cdot\|$ and let $E$ be a dense subspace of $X$. Assume that $J$ is a continuous functional defined on $X$ which is E-differentiable. Suppose that $J$ satisfies the following conditions.

$\left(\mathbf{A}_{\mathbf{1}}\right) J$ is an even functional, i.e., $J(-u)=J(u)$ for every $u \in X$, and it is bounded from below.

$\left(\mathbf{A}_{\mathbf{2}}\right)$ If $u \in X,\left\{u_{n}\right\} \subset X,\left|J^{\prime}\left(u_{n}\right)\right| \rightarrow 0$, and $u_{n} \rightarrow u$ as $n \rightarrow \infty$, then

$$
\left|J^{\prime}(u)\right|=0 .
$$

$\left(\mathbf{A}_{\mathbf{3}}\right) J$ is weakly sequentially lower semicontinuous. 
(A $\left.\mathbf{A}_{4}\right)$ The set $\{u \in X \mid J(u) \leq J(0)\}$ is bounded in $X$.

$\left(\mathbf{A}_{\mathbf{5}}\right)$ For any positive integer $k$, there exists a $k$-dimensional subspace $X_{k}$ of $X$ and $\rho_{k}>0$ such that $\sup _{X_{k} \cap S_{\rho_{k}}} J<J(0)$, where $S_{\rho}=\{u \in X \mid\|u\|=\rho\}$. Then, $J$ has infinitely many pairs of critical points $\left\{ \pm u_{k} \mid k=1,2, \ldots\right\}$ satisfying

$$
J\left( \pm u_{k}\right) \leq J(0), \quad u_{k} \neq 0 \text { for } k=1,2, \ldots, \quad \text { and } u_{k} \rightarrow 0 \quad \text { as } k \rightarrow \infty .
$$

Remark 1.2. We will assume, without loss of generality, $J(0)=0$.

Remark 1.3. The assumption $\left(\mathbf{A}_{\mathbf{2}}\right)$ can be deduced from the following assumption. $\left(\mathbf{A}_{2}^{\prime}\right)$ If $\left\{u_{n}\right\} \subset X, u \in X$, and $u_{n} \rightarrow u$ in $X$, then

$$
\left\langle J^{\prime}\left(u_{n}\right), h\right\rangle \rightarrow\left\langle J^{\prime}(u), h\right\rangle \quad \forall h \in E .
$$

Therefore, the result of Theorem 1.1 is true if the assumption $\left(\mathbf{A}_{\mathbf{2}}\right)$ is replaced by $\left(\mathbf{A}_{2}^{\prime}\right)$.

Obviously, either condition $\left(\mathbf{A}_{\mathbf{2}}\right)$ or $\left(\mathbf{A}_{\mathbf{2}}^{\prime}\right)$ is weaker than the Palais-Smale condition for nonsmooth functionals (see [21, Definition 3.8]). Concerning various differential equations, there are many cases in which the functionals satisfy $\left(\mathbf{A}_{\mathbf{1}}\right)-\left(\mathbf{A}_{\mathbf{5}}\right)$, but do not satisfy the Palais-Smale condition. Using a new genus with respect to the weak topology of $X$ and the method of descending flow, we will give the proof of Theorem 1.1 in the following three sections.

In the literature, intensive work was carried out in the 1990s on critical point theory for nonsmooth functionals. We discuss and compare some related work here. Corvellec, Degiovanni, and Marzocchi in [9] and Degiovanni and Marzocchi in [13] gave the following definition of the weak slope for a continuous functional defined in a metric space.

Definition 1.4 (Definition 2.1 of [9] or Definition 2.1 of [13]). Let $Y$ be a metric space endowed with the metric $d$. Let $f: Y \rightarrow \mathbb{R}$ be a continuous function and $u \in Y$. We denote by $|d f|(u)$ the supremum of the $\sigma$ 's in $[0,+\infty]$ such that there exist $\delta>0$ and $\mathcal{H}: B(u, \delta) \times[0, \delta] \rightarrow Y$ continuous with

$$
d(\mathcal{H}(v, t), v) \leq t \quad \text { and } \quad f(\mathcal{H}(v, t)) \leq f(v)-\sigma t,
$$

where $B(u, \delta)=\{v \in Y \mid d(u, v)<\delta\}$. The extended real number $|d f|(u)$ is called the weak slope of $f$ at $u$, and $u$ is called a critical point of $f$ if $|d f|(u)=0$.

Using this, the authors of [9] also gave the definition of the weak slope for a lower semicontinuous functional $f$ defined in the metric space $Y$. More precisely, let $Y$ be a metric space endowed with the metric $d$ and $f: Y \rightarrow \mathbb{R} \cup\{+\infty\}$ be a lower semicontinuous function. Let $\mathcal{D}(f)=\{u \in Y \mid f(u)<+\infty\}$, epi $(f)=\{(u, \xi) \in$ $Y \times \mathbb{R} \mid f(u) \leq \xi\}$, and

$$
\mathcal{G}_{f}: \operatorname{epi}(f) \rightarrow \mathbb{R}, \quad \mathcal{G}_{f}(u, \xi)=\xi .
$$

The set $Y \times \mathbb{R}$ is endowed with the metric $\varrho((u, \xi),(v, \mu))=\left(d^{2}(u, v)+(\xi-\mu)^{2}\right)^{1 / 2}$ and epi $(f)$ with the induced metric.

Definition 1.5 (Definition 2.6 of [9] or Definition 2.4 of [13]). Let $Y$ be a metric space endowed with the metric d. Let $f: Y \rightarrow \mathbb{R} \cup\{+\infty\}$ be a lower semicontinuous function and $u \in \mathcal{D}(f)$. The weak slope of $f$ at $u$ is defined by

$$
|d f|(u)= \begin{cases}\frac{\left|d \mathcal{G}_{f}\right|(u, f(u))}{\sqrt{1-\left(\left|d \mathcal{G}_{f}\right|(u, f(u))\right)^{2}}} & \text { if }\left|d \mathcal{G}_{f}\right|(u, f(u))<1 \\ +\infty & \text { if }\left|d \mathcal{G}_{f}\right|(u, f(u))=1\end{cases}
$$

Copyright (c) by SIAM. Unauthorized reproduction of this article is prohibited. 
$u \in \mathcal{D}(f)$ is called a critical point of $f$ if $|d f|(u)=0$.

The idea of Definition 1.5 is to reduce the study of the lower semicontinuous function $f$ to that of the continuous function $\mathcal{G}_{f}$. In view of critical point theory, it is necessary to introduce a condition which implies a bijective correspondence between the critical points of $f$ and those of $\mathcal{G}_{f}$.

Definition 1.6 (Definition 2.2 of [15] or (4.1) of [9]). We say that $f$ satisfies the condition epi on $Y$ if, for any $b>0$,

$$
\inf \left\{\left|d \mathcal{G}_{f}\right|(u, \xi)|u \in \mathcal{D}(f), f(u)<\xi,| \xi \mid \leq b\right\}>0 .
$$

The epi condition guarantees that a critical point of $\mathcal{G}_{f}$ corresponds to a critical point of the original functional $f$. Therefore, using the weak slope to obtain critical points of a lower semicontinuous functional, the epi condition must be verified.

From [4, Theorem 1.1.2], it is easy to see that if $J$ is $E$-differentiable, then $\left|J^{\prime}(u)\right| \leq|d J|(u)$ for any $u \in X$.

Based on the weak slope, the authors of [9] and [13] established some deformation theorems and then developed a nonsmooth critical point theory for lower semicontinuous functionals. This theory has many important applications; see, for example, $[4,14,15]$. In particular, in [15] Degiovanni and Schuricht obtained a Clark-type theorem for lower semicontinuous functionals defined in metric spaces (see [15, Theorem 2.5]). The following theorem is a direct consequence of [15, Theorem 2.5].

THEOREM 1.7. Let $Y$ be a complete metric space which is a subset of some linear space $X$ in the set-theoretic sense and for which $0 \in Y$ and $-u \in Y$ whenever $u \in Y$. Assume that $J: Y \rightarrow \mathbb{R} \cup\{+\infty\}$ is a lower semicontinuous functional defined on $Y$. Suppose that $J$ satisfies the following conditions.

(i) $J$ is an even functional, i.e., $J(-u)=J(u)$ for every $u \in Y$, and it is bounded from below.

(ii) $J$ satisfies the epi condition.

(iii) For any $c<J(0)$, the function $J$ satisfies the condition $(P S)_{c}$.

(iv) For any positive integer $k$, there exists a continuous odd map $\psi$ from the $k$-dimensional sphere $S^{k}$ to $Y$ such that

$$
\sup _{u \in S^{k}} J(\psi(u))<J(0) .
$$

Then, $J$ has infinitely many pairs of critical points $\left\{ \pm u_{k} \mid k=1,2, \ldots\right\}$ satisfying

$$
J\left( \pm u_{k}\right)<J(0) \quad \text { for } k=1,2, \ldots
$$

We point out that Theorem 1.1 cannot be derived from the above result. The reason for this is that, under the weak topology of a separable and reflexive Banach space, it is very difficult to verify the epi condition for a lower semicontinuous functional. More precisely, let $X$ be a separable and reflexive Banach space and $J$ the functional satisfying the conditions $\left(\mathbf{A}_{\mathbf{1}}\right)-\left(\mathbf{A}_{\mathbf{5}}\right)$ in Theorem 1.1. Because $X$ is a separable and reflexive Banach space and $J$ satisfies the conditions $\left(\mathbf{A}_{\mathbf{3}}\right)$ and $\left(\mathbf{A}_{\mathbf{4}}\right)$, if we choose $Y=\{u \in X \mid J(u) \leq J(0)\}$ endowed with the weak topology of $X$, then $Y$ is a compact metric space (see Lemma 3.1 in section 3). Now the functional $J$ in Theorem 1.1 is merely a lower semicontinuous functional in $Y$ and not a continuous functional in $Y$ in general. Because $J$ satisfies the conditions $\left(\mathbf{A}_{\mathbf{1}}\right)-\left(\mathbf{A}_{\mathbf{5}}\right)$ in Theorem 1.1 , it is easy to verify that the functional $J$ in Theorem 1.1 satisfies the conditions (i), (iii), and (iv) in Theorem 1.7. In particular, we must point out that $J$ satisfies 
the $(P S)_{c}$ condition, i.e., the condition (iii) in Theorem 1.7, since $Y$ is a compact metric space. However, the conditions $\left(\mathbf{A}_{\mathbf{1}}\right)-\left(\mathbf{A}_{\mathbf{5}}\right)$ in Theorem 1.1 cannot imply that the functional $J$ satisfies the condition (ii), i.e., the epi condition. To the best of our knowledge, under the weak topology of $X$, the epi condition is only verified for the functional $f: X \rightarrow \mathbb{R}$ with a very special form: $f=f_{0}+f_{1}$, where $f_{0}: X \rightarrow \mathbb{R}$ is a $C^{1}$, convex, and weakly lower semicontinuous functional, $f_{1}: X \rightarrow \mathbb{R}$ is of class $C^{1}$, and $f_{1}^{\prime}: X \rightarrow X^{\prime}$ is completely continuous (see [12, Proposition 5.2]). It would be a very interesting question to pursue in the future whether a condition like $\left(\mathbf{A}_{\mathbf{2}}\right)$ could replace the $P S$ condition so that it is incorporated into the critical point theory developed by Degiovanni and his collaborators.

As an application of Theorem 1.1, in section 5 we consider the following quasilinear elliptic equation:

(1.1) $-\operatorname{div}\left(D_{\xi} \Psi(x, u, \nabla u)\right)+D_{s} \Psi(x, u, \nabla u)=K(x)|u|^{q-2} u \quad$ in $\Omega, \quad u=0 \quad$ on $\partial \Omega$,

where $\Omega$ is a smooth bounded domain in $\mathbb{R}^{N}(N \geq 2)$. The conditions on the function $\Psi: \Omega \times \mathbb{R} \times \mathbb{R}^{N} \rightarrow \mathbb{R},(x, s, \xi) \mapsto \Psi(x, s, \xi)$ will allow the quasi-linear elliptic operator

$$
-\operatorname{div}\left(D_{\xi} \Psi(x, u, \nabla u)\right)+D_{s} \Psi(x, u, \nabla u)
$$

to be very degenerate. In fact, under our assumptions on $\Psi, \Psi(x, s, \cdot)$ may vanish in an open set containing $\xi=0$ in $\mathbb{R}^{N}$ for any $(x, s)$ (see the example (5.7) in section 5), and $\Psi$ may be not strictly convex with respect to $\xi$. Therefore, the Euler-Lagrange functional corresponding to (1.1) does not satisfy the Palais-Smale condition. The very degenerate-type elliptic operators involved in (1.1) are related to some traffic congestion dynamic models $[3,8]$. In section 5 , using Theorem 1.1, we prove that this equation has infinitely many solutions. In a sense, our result improves the previous results on quasi-linear elliptic equations in [11, 22].

As another application of Theorem 1.1, in section 6 we consider the existence of homoclinic orbits for the following second-order Hamiltonian system:

$$
\ddot{u}+\nabla_{u} V(t, u)=0,
$$

where $V$ is even with respect to $u, \nabla_{u} V$ exists, $\nabla_{u} V \in C\left(\mathbb{R} \times \mathbb{R}^{n}, \mathbb{R}^{n}\right), V(t, u)=$ $-K(t, u)+W(t, u), W$ is sublinear with respect to $u$, and $K$ only satisfies the "pinching" condition [17]

$$
\tau_{1}|u|^{2} \leq K(t, u) \leq \tau_{2}|u|^{2}
$$

for some constants $\tau_{2}>\tau_{1}>0$. Under these assumptions, the Euler-Lagrange functional corresponding to $(1.2)$ is not $C^{1}$ in the Sobolev space $H^{1}(\mathbb{R})$ and does not satisfy the Palais-Smale condition in general. Using Theorem 1.1, we prove that this equation has infinitely many homoclinic orbits. Our result improves the previous results in $[17,24,27]$.

2. The genus with respect to the weak topology and its properties. Let $X$ be a separable and reflexive Banach space with norm $\|\cdot\|$ and let $X^{\prime}$ be its dual space. Let $\left\{\phi_{k}\right\}$ be a sequence dense in the unit ball of $X^{\prime}$ and define as in [12], for every $u, v \in X$,

$$
(u, v)_{w}=\sum_{k=1}^{\infty} \frac{1}{2^{k}} \phi_{k}(u) \phi_{k}(v) .
$$

Copyright (c) by SIAM. Unauthorized reproduction of this article is prohibited. 
Then $(\cdot, \cdot)_{w}$ is a scalar product on $X$ whose associated norm $\|\cdot\|_{w}$ satisfies

$$
\|u\|_{w} \leq\|u\| \quad \forall u \in X
$$

and induces the weak topology of $X$ on every $\|\cdot\|$-bounded subset of $X$. We denote the normed space $\left(X ;\|\cdot\|_{w}\right)$ by $X_{w}$. It is easy to see that if $\left\{u_{n}\right\} \subset X$ and $\left\{\left\|u_{n}\right\|\right\}$ is bounded, then

$$
u_{n} \rightarrow u \Leftrightarrow\left\|u_{n}-u\right\|_{w} \rightarrow 0, \quad n \rightarrow \infty .
$$

The topology generated by $\|\cdot\|_{w}$ is denoted by $w$, and all topological notations related to $\|\cdot\|_{w}$ will include this symbol.

Let $S(X)$ be the class of subsets of $X \backslash\{0\}$ symmetric with respect to the origin and closed with respect to the $\|\cdot\|$-topology. Recall that the genus of $A \in S(X)$, denoted by $\gamma(A)$, is the least integer $n$ such that there exists an odd and continuous $\operatorname{map} \phi: A \rightarrow \mathbb{R}^{n} \backslash\{0\}$.

Definition 2.1. Let $S\left(X_{w}\right)$ be the class of subsets of $X \backslash\{0\}$ symmetric with respect to the origin and closed with respect to the $w$-topology. If $A \in S\left(X_{w}\right)$, the weak genus of $A$, denoted by $\gamma_{w}(A)$, is the least integer $m$ such that there exists an odd and $w$-continuous map $\psi: A \rightarrow \mathbb{R}^{m} \backslash\{0\}$.

Remark 2.2. Since a closed set in $X_{w}$ is also a closed set in the Banach space $X$, we deduce that $S\left(X_{w}\right) \subset S(X)$ and, for any $A \in S\left(X_{w}\right)$,

$$
\gamma(A) \leq \gamma_{w}(A) .
$$

Remark 2.3. Assume $x_{0} \in X$ and $\left\|x_{0}\right\|>1$. Let $A=\left\{x \mid\left\|x-x_{0}\right\|=1\right\} \cup$ $\left\{x \mid\left\|x+x_{0}\right\|=1\right\}$. Then $A \in S(X)$, but $A \notin S\left(X_{w}\right)$.

Remark 2.4. Let $X$ be an infinite-dimensional and separable Hilbert space and $\left\{e_{n}\right\}$ an orthonormal base of $X$. Define

$$
L=\bigcup_{0 \leq t \leq 1}\left(\left\{(1-2 t) e_{1}+t e_{2}\right\} \bigcup\left(\bigcup_{n=2}^{\infty}\left\{(1-t) e_{n}+t e_{n+1}-e_{1}\right\}\right)\right)
$$

Then $L$ is closed with respect to the $\|\cdot\|$-topology, but is not closed with respect to the $w$-topology. Indeed, $c l_{w}(L)=L \cup\left\{-e_{1}\right\}$, where $c l_{w}(L)$ denotes the closure of $L$ in $X_{w}$. Let $A=L \cup(-L)$, where $-L=\{u \mid-u \in L\}$. Then $A \in S\left(X_{w}\right)$. From the construction of $A$ we see that

$$
\gamma(A)=1, \quad \gamma_{w}(A)=2 .
$$

Therefore, $\gamma$ and $\gamma_{w}$ do not coincide on $S\left(X_{w}\right)$.

For the properties of the genus $\gamma$, one may consult [7] and [6]. Our following proposition shows that the weak genus $\gamma_{w}$ and the genus $\gamma$ share similar properties. The proof of the properties of $\gamma_{w}$ is similar to the proof of the properties of $\gamma$, and one may consult p. 17 of [7].

Proposition 2.5. The weak genus $\gamma_{w}$ has the following properties.

(i) If $A_{1}, A_{2} \in S\left(X_{w}\right)$ and if there exists an odd and $w$-continuous map $f: A_{1} \rightarrow$ $A_{2}$, then $\gamma_{w}\left(A_{1}\right) \leq \gamma_{w}\left(A_{2}\right)$. In particular, if $A_{1} \subset A_{2}$, then $\gamma_{w}\left(A_{1}\right) \leq \gamma_{w}\left(A_{2}\right)$.

(ii) For $A_{1}, A_{2} \in S\left(X_{w}\right), \gamma_{w}\left(A_{1} \cup A_{2}\right) \leq \gamma_{w}\left(A_{1}\right)+\gamma_{w}\left(A_{2}\right)$. 
(iii) If $A \in S\left(X_{w}\right)$ and $A$ is w-compact, then $\gamma_{w}(A)<+\infty$ and $A$ has a neighborhood $U$ open with respect to the $w$-topology such that $c l_{w}(U) \in S\left(X_{w}\right)$ and $\gamma_{w}\left(c l_{w}(U)\right)=\gamma_{w}(A)$.

(iv) If $G \in S\left(X_{w}\right)$ and if there exists an odd homeomorphism of the $n$-sphere onto $G$, then $\gamma_{w}(G)=n+1$.

(v) If $\left\{G_{n}\right\}$ is a decreasing sequence of w-compact sets belonging to $S\left(X_{w}\right)$ and if $G=\cap_{n=1}^{\infty} G_{n}$, then $G \in S\left(X_{w}\right)$ and

$$
\gamma_{w}(G)=\lim _{n \rightarrow \infty} \gamma_{w}\left(G_{n}\right) .
$$

Remark 2.6. There is no ambiguity regarding homeomorphism in Proposition 2.5 (iv), since $\psi: G \rightarrow S^{n}$ is a homeomorphism with respect to the $\|\cdot\|$-topology if and only if it is a homeomorphism with respect to the $w$-topology. This is easily seen via the compactness of $S^{n}$.

3. A gradient field and the related descending flow. Throughout this section, let $X$ be a separable and reflexive Banach space with norm $\|\cdot\|, E$ a dense subspace of $X$, and $J: X \rightarrow \mathbb{R}$ an $E$-differentiable functional. For any $c \in \mathbb{R}$, set

$$
J^{c}=\{u \in X \mid J(u) \leq c\} .
$$

Lemma 3.1. If $J$ satisfies the conditions $\left(\mathbf{A}_{\mathbf{3}}\right)$ and $\left(\mathbf{A}_{\mathbf{4}}\right)$ and $c \leq 0$, then $J^{c}$ is w-compact.

Proof. We may assume $J^{c} \neq \emptyset$. Since $c \leq 0, J^{c}$ is bounded in $X$ by $\left(\mathbf{A}_{\mathbf{4}}\right)$. Let $\left\{u_{n}\right\} \subset J^{c}$. Then $\left\{\left\|u_{n}\right\|\right\}$ is bounded. Since $X$ is a reflexive Banach space, there exists $u \in X$ such that, up to a subsequence, $u_{n} \rightarrow u$. Since $J$ is weakly sequentially lower semicontinuous, we have $\liminf _{n \rightarrow \infty} J\left(u_{n}\right) \geq J(u)$. It follows that $u \in J^{c}$. Therefore, $J^{c}$ is $w$-compact.

Let

$$
\mathcal{K}=\left\{u \in X|J(u) \leq 0,| J^{\prime}(u) \mid=0\right\} .
$$

If $J$ is an even functional, then $0 \in \mathcal{K}$.

For $c \leq 0$, let

$$
\mathcal{K}^{c}=\mathcal{K} \cap J^{c}=\left\{u \in X|J(u) \leq c,| J^{\prime}(u) \mid=0\right\} .
$$

Lemma 3.2. If $J$ satisfies the conditions $\left(\mathbf{A}_{\mathbf{2}}\right)-\left(\mathbf{A}_{\mathbf{4}}\right)$ and $c \leq 0$, then $\mathcal{K}^{c}$ is $w$ compact.

Proof. We may assume $\mathcal{K}^{c} \neq \emptyset$. By Lemma 3.1, we only need to prove that $\mathcal{K}^{c}$ is $w$-closed in $J^{c}$. Let $\left\{u_{n}\right\} \subset \mathcal{K}^{c}$. The proof of Lemma 3.1 shows that $u_{n} \rightarrow u$ for some $u \in J^{c}$. Since $\left|J^{\prime}\left(u_{n}\right)\right|=0$, the condition $\left(\mathbf{A}_{\mathbf{2}}\right)$ implies that $\left|J^{\prime}(u)\right|=0$. It follows that $u \in \mathcal{K}^{c}$. Therefore, $\mathcal{K}^{c}$ is a $w$-compact set.

Lemma 3.3. If $J$ satisfies the conditions $\left(\mathbf{A}_{\mathbf{2}}\right)-\left(\mathbf{A}_{\mathbf{4}}\right), V$ is a w-open neighborhood of $\mathcal{K}$, and $J^{0} \backslash V \neq \emptyset$, then

$$
\inf \left\{\left|J^{\prime}(u)\right| \mid u \in J^{0} \backslash V\right\}>0 .
$$

Proof. If (3.4) were false, then there would be $u_{n} \in J^{0} \backslash V$ such that $\left|J^{\prime}\left(u_{n}\right)\right| \rightarrow 0$. By Lemma 3.1, there exists $u \in J^{0} \backslash V$ such that, up to a subsequence, $u_{n} \rightarrow u$. By $\left(\mathbf{\mathbf { A } _ { 2 }}\right)$, we have $\left|J^{\prime}(u)\right|=0$, which is a contradiction. 
To prove Theorem 1.1, we will use an argument of contradiction. For this, we assume that there exists $\iota>0$ such that

$$
\mathcal{K} \cap B_{X_{w}}(0, \iota)=\{0\},
$$

where

$$
B_{X_{w}}(u, r)=\left\{v \in X_{w} \mid\|v-u\|_{w}<r\right\}
$$

Let

$$
\mathcal{K}^{\prime}=\mathcal{K} \backslash\{0\} .
$$

Then, by (3.5) and Lemma $3.2, \mathcal{K}^{\prime}$ is a $w$-compact set and

$$
d_{w}\left(0, \mathcal{K}^{\prime}\right) \geq \iota
$$

where

$$
d_{w}(u, A)=\inf _{v \in A}\|u-v\|_{w}
$$

For $r>0$, let

$$
\left(\mathcal{K}^{\prime}\right)_{r}=\left\{u \in X \mid d_{w}\left(u, \mathcal{K}^{\prime}\right)<r\right\} .
$$

Lemma 3.4. Assume that $J$ satisfies the conditions $\left(\mathbf{A}_{\mathbf{1}}\right)-\left(\mathbf{A}_{\mathbf{4}}\right)$ and suppose that (3.7) holds. Then there exists

$$
\zeta: \tilde{X} \rightarrow E \backslash\{0\},
$$

where $\tilde{X}:=\left\{u \in X|| J^{\prime}(u) \mid \neq 0, J(u)<0\right\}$, satisfying the following.

(i) $\zeta$ is an equivariant locally Lipschitz mapping, i.e., for any $u \in \tilde{X}, \zeta(-u)=$ $-\zeta(u)$ and there exist $L_{u}>0$ and $r_{u}>0$ such that for any $v_{1}, v_{2} \in B_{X}\left(u, r_{u}\right)$,

$$
\left\|\zeta\left(v_{1}\right)-\zeta\left(v_{2}\right)\right\| \leq L_{u}\left\|v_{1}-v_{2}\right\|,
$$

where $B_{X}(u, r)=\{v \in X \mid\|v-u\|<r\}$.

(ii) For any $u \in \tilde{X},\|\zeta(u)\| \leq 1$ and $\left\langle J^{\prime}(u), \zeta(u)\right\rangle>0$.

(iii) If $c<0$ and $J^{c} \backslash\left(\mathcal{K}^{\prime}\right)_{\iota / 20} \neq \emptyset$, then

$$
\inf \left\{\left\langle J^{\prime}(u), \zeta(u)\right\rangle \mid u \in J^{c} \backslash\left(\mathcal{K}^{\prime}\right)_{\iota / 20}\right\}>0 .
$$

(iv) If $\left\{u \in X \mid J(u)<0, u \in \operatorname{cl}_{w}\left(\left(\mathcal{K}^{\prime}\right)_{\iota / 3} \backslash\left(\mathcal{K}^{\prime}\right)_{\iota / 4}\right)\right\} \neq \emptyset$, then

$$
\inf \left\{\left\langle J^{\prime}(u), \zeta(u)\right\rangle \mid J(u)<0, u \in \operatorname{cl}_{w}\left(\left(\mathcal{K}^{\prime}\right)_{\iota / 3} \backslash\left(\mathcal{K}^{\prime}\right)_{\iota / 4}\right)\right\}>0 .
$$

Proof. For any $u \in \tilde{X}$, by the definition of $\left|J^{\prime}(u)\right|$, there exists $h_{u} \in E$ satisfying

$$
\left\|h_{u}\right\|=1
$$

and

$$
\left\langle J^{\prime}(u), h_{u}\right\rangle \geq \frac{3}{4} \min \left\{\left|J^{\prime}(u)\right|, 1\right\} .
$$

From the definition of $E$-differentiability and the fact that $J$ is continuous in $X$, there exists $\delta_{u}$ such that

$$
0<\delta_{u}<\iota / 60
$$

Copyright $@$ by SIAM. Unauthorized reproduction of this article is prohibited. 


$$
0>\sup _{v \in B_{X}\left(u, \delta_{u}\right)} J(v) \geq \inf _{v \in B_{X}\left(u, \delta_{u}\right)} J(v)>\frac{3}{2} J(u)
$$

and

$$
\left\langle J^{\prime}(v), h_{u}\right\rangle \geq \frac{1}{2} \min \left\{\left|J^{\prime}(u)\right|, 1\right\}>0 \quad \text { if } v \in B_{X}\left(u, \delta_{u}\right) .
$$

Then

$$
\mathcal{A}=\left\{B_{X}\left(u, \delta_{u}\right)|| J^{\prime}(u) \mid \neq 0, J(u)<0\right\}
$$

is an open covering of $\tilde{X}$.

Since $\tilde{X}$ is a metric space and hence a paracompact space, there exists a locally finite open covering $\mathcal{M}=\left\{M_{i} \mid i \in \Lambda\right\}$ of $\tilde{X}$ finer than $\mathcal{A}$. Define

$$
\lambda_{i}(u)=\frac{d\left(u, X \backslash M_{i}\right)}{\sum_{j \in \Lambda} d\left(u, X \backslash M_{j}\right)}, \quad u \in \tilde{X},
$$

where

$$
d(u, A)=\inf _{v \in A}\|u-v\| .
$$

Since each $u \in \tilde{X}$ has a neighborhood intersecting only finitely many $M_{j}, \sum_{j \in \Lambda} d(u, X \backslash$ $M_{j}$ ) is only a finite sum, and each $\lambda_{i}$ is a Lipschitz continuous functional on $\tilde{X}$ with respect to the $\|\cdot\|$ norm.

For any $i \in \Lambda$, choose one $u_{i} \in \tilde{X}$ such that $M_{i} \subset B_{X}\left(u_{i}, \delta_{u_{i}}\right)$. Define

$$
\zeta^{*}(u):=\sum_{i \in \Lambda} \lambda_{i}(u) h_{u_{i}} \in E, \quad u \in \tilde{X}
$$

and

$$
\zeta(u):=\frac{1}{2}\left(\zeta^{*}(u)-\zeta^{*}(-u)\right), \quad u \in \tilde{X} .
$$

For every $u \in \tilde{X}$, the sum in (3.14) is only a finite sum. Clearly, $\zeta$ satisfies (i). Since $J$ is an even functional, we have

$$
\left\langle J^{\prime}(-u), h\right\rangle=\left\langle-J^{\prime}(u), h\right\rangle \quad \forall u \in X \forall h \in E .
$$

Then by (3.10)-(3.16), we deduce that $\zeta$ satisfies (ii).

Now we prove (iii). For $u \in J^{c} \backslash\left(\mathcal{K}^{\prime}\right)_{\iota / 20}$, there exist only a finite number of elements from $\mathcal{M}$, denoted by $M_{1}, M_{2}, \ldots, M_{k}$, such that for all $1 \leq i \leq k$, either $u \in M_{i}$ or $-u \in M_{i}$. Then

$$
\zeta(u)=\frac{1}{2} \sum_{i=1}^{k} \lambda_{i}(u) h_{u_{i}}-\frac{1}{2} \sum_{i=1}^{k} \lambda_{i}(-u) h_{u_{i}} .
$$

Clearly, $\pm u \in M_{i}$ if and only if $\lambda_{i}( \pm u) \neq 0$, in which case $\pm u \in B_{X}\left(u_{i}, \delta_{u_{i}}\right)$ and

$$
\left\langle J^{\prime}( \pm u), h_{u_{i}}\right\rangle \geq \frac{1}{2} \min \left\{\left|J^{\prime}\left(u_{i}\right)\right|, 1\right\} .
$$

We first consider the case $u \in M_{i}$. Since $J(u) \leq c$, by (3.12), we have

$$
J\left(u_{i}\right)<\frac{2}{3} c .
$$

Copyright (c) by SIAM. Unauthorized reproduction of this article is prohibited. 
Since $u \in B_{X}\left(u_{i}, \delta_{u_{i}}\right)$ and $u \notin\left(\mathcal{K}^{\prime}\right)_{\iota / 20}$, by $(3.11)$, we see that

$$
u_{i} \notin\left(\mathcal{K}^{\prime}\right)_{\iota / 40} \text {. }
$$

Combining (3.19) and (3.20) yields

$$
u_{i} \in J^{\frac{2}{3} c} \backslash\left(\mathcal{K}^{\prime}\right)_{\iota / 40} .
$$

In the case $-u \in M_{i}$, the same argument from (3.19) to (3.21), with $-u$ instead of $u$, holds. Since $\frac{2}{3} c<0$, by Lemma 3.3 ,

$$
\left|J^{\prime}\left(u_{i}\right)\right| \geq m_{c}:=\inf \left\{\left|J^{\prime}(u)\right| \mid u \in J^{\frac{2}{3} c} \backslash\left(\mathcal{K}^{\prime}\right)_{\iota / 40}\right\}>0 .
$$

By (3.17), (3.18), and (3.22), we have

$$
\begin{aligned}
\left\langle J^{\prime}(u), \zeta(u)\right\rangle & =\frac{1}{2} \sum_{i=1}^{k} \lambda_{i}(u)\left\langle J^{\prime}(u), h_{u_{i}}\right\rangle-\frac{1}{2} \sum_{i=1}^{k} \lambda_{i}(-u)\left\langle J^{\prime}(u), h_{u_{i}}\right\rangle \\
& =\frac{1}{2} \sum_{i=1}^{k} \lambda_{i}(u)\left\langle J^{\prime}(u), h_{u_{i}}\right\rangle+\frac{1}{2} \sum_{i=1}^{k} \lambda_{i}(-u)\left\langle J^{\prime}(-u), h_{u_{i}}\right\rangle \\
& \geq \frac{1}{4} \min \left\{1,\left|J^{\prime}\left(u_{i}\right)\right|, i=1,2, \ldots, k\right\}\left(\sum_{i=1}^{k} \lambda_{i}(u)+\sum_{i=1}^{k} \lambda_{i}(-u)\right) \\
& =\frac{1}{2} \min \left\{1,\left|J^{\prime}\left(u_{i}\right)\right|, i=1,2, \ldots, k\right\} \geq \min \left\{1, m_{c}\right\} / 2>0 .
\end{aligned}
$$

This implies (iii).

It suffices to prove (iv). Assume $J(u)<0$ and $u \in c l_{w}\left(\left(\mathcal{K}^{\prime}\right)_{\iota / 3} \backslash\left(\mathcal{K}^{\prime}\right)_{\iota / 4}\right)$. As in the proof of (iii), there exist finite $u_{i} \in \tilde{X}, 1 \leq i \leq k$, such that (3.17) and (3.18) hold. For any $i$, since either $u \in B_{X}\left(u_{i}, \delta_{u_{i}}\right)$ or $-u \in B_{X}\left(u_{i}, \delta_{u_{i}}\right)$ and since $u \in c l_{w}\left(\left(\mathcal{K}^{\prime}\right)_{\iota / 3} \backslash\left(\mathcal{K}^{\prime}\right)_{\iota / 4}\right),(3.11)$ and $(3.7)$ can be used to deduce that

$$
u_{i} \in J^{0} \backslash\left(B_{X_{w}}(0, \iota / 5) \cup\left(\mathcal{K}^{\prime}\right)_{\iota / 5}\right) .
$$

This, together with Lemma 3.3, implies

$$
\left|J^{\prime}\left(u_{i}\right)\right| \geq m_{*}:=\inf \left\{\left|J^{\prime}(u)\right| \mid J(u) \leq 0, u \notin B_{X_{w}}(0, \iota / 5) \cup\left(\mathcal{K}^{\prime}\right)_{\iota / 5}\right\}>0 .
$$

We then conclude as (3.23).

Set $A=\left(\mathcal{K}^{\prime}\right)_{\iota / 40}$ and $B=X \backslash\left(\mathcal{K}^{\prime}\right)_{\iota / 20}$, and define

$$
\varrho(u)=\frac{d_{w}(u, A)}{d_{w}(u, A)+d_{w}(u, B)}, \quad u \in X .
$$

Then $\varrho$ is an even functional which is Lipschitz continuous with respect to the $\|\cdot\|_{w^{-}}$ norm, $\left.\varrho\right|_{A}=0,\left.\varrho\right|_{B}=1$, and $0 \leq \varrho \leq 1$. The fact that $\|v\|_{w} \leq\|v\|$ for $v \in X$ implies that $\varrho$ is also Lipschitz continuous with respect to the $\|\cdot\|$-norm. Let

$$
\chi(u)=-\varrho(u) \zeta(u), \quad u \in \tilde{X} .
$$

The conclusion (i) in Lemma 3.4 implies that $\chi$ is Lipschitz continuous with respect to the $\|\cdot\|$-norm. Therefore, the initial value problem

$$
\left\{\begin{array}{l}
\frac{d \eta}{d t}=\chi(\eta) \\
\eta(0, u)=u \in \hat{X}:=\{v \in X \mid J(v)<0\}
\end{array}\right.
$$

Copyright (c) by SIAM. Unauthorized reproduction of this article is prohibited. 
has a unique solution in $\hat{X}$, denoted by $\eta(t, u)$, with right maximal interval of existence $[0, T(u))$. The conclusion (ii) in Lemma 3.4, together with the construction of $\chi$, implies that $\left\langle J^{\prime}(u), \chi(u)\right\rangle \leq 0$ for all $u \in \hat{X}$. This fact, together with the equation

$$
\frac{d}{d t} J(\eta(t, u))=\left\langle J^{\prime}(\eta(t, u)), \chi(\eta(t, u))\right\rangle,
$$

implies that $J$ is nonincreasing along trajectories of $\eta$. Therefore, $\hat{X}$ is an invariant set of the flow $\eta$. The conclusion (ii) in Lemma 3.4, together with the construction of $\chi$, also implies $\|\chi(u)\| \leq 1$ for all $u \in \hat{X}$. From this and the fact that $\hat{X}$ is an invariant set of the flow $\eta$, we see that $T(u)=+\infty$ for any $u \in \hat{X}$. From the conclusion (i) in Lemma 3.4 and the definition of $\chi$, we have $\chi(-u)=-\chi(u)$ for all $u \in \hat{X}$. It follows that, for any $(t, u) \in[0,+\infty) \times \hat{X}$,

$$
\eta(t,-u)=-\eta(t, u) .
$$

Lemma 3.5. Assume $J$ satisfies the conditions $\left(\mathbf{A}_{\mathbf{1}}\right)-\left(\mathbf{A}_{\mathbf{4}}\right)$ and $\inf _{X} J<0$. Suppose that (3.7) holds. Then there exists $r_{0}>0$ satisfying that, for any $c<0$, there exists $T_{c}>0$ such that for any $u \in J^{c}$,

$$
\left\|\eta\left(T_{c}, u\right)\right\|_{w} \geq r_{0} .
$$

Proof. Arguing indirectly, assume that, for any $0<r<\iota / 6$, there exist $c_{r}<0$ and a sequence $\left\{u_{n}\right\} \subset J^{c_{r}}$ and $T_{n} \rightarrow+\infty$ such that

$$
\left\|\eta\left(T_{n}, u_{n}\right)\right\|_{w}<r .
$$

We shall prove that, if $n$ is large enough, then there exists $0<t_{n}<T_{n}$ such that

$$
\eta\left(t_{n}, u_{n}\right) \in\left(\mathcal{K}^{\prime}\right)_{\iota / 4}
$$

If this were not true, then, up to a subsequence,

$$
\eta\left(\left[0, T_{n}\right], u_{n}\right) \subset J^{c_{r}} \backslash\left(\mathcal{K}^{\prime}\right)_{\iota / 4}, n=1,2, \ldots
$$

By the conclusion (iii) in Lemma 3.4, we have

$$
a_{r}:=\inf \left\{\left\langle J^{\prime}(u), \zeta(u)\right\rangle \mid u \in J^{c_{r}} \backslash\left(\mathcal{K}^{\prime}\right)_{\iota / 4}\right\}>0 .
$$

It follows that as $n \rightarrow \infty$,

$$
\begin{aligned}
J\left(\eta\left(T_{n}, u_{n}\right)\right)-J\left(u_{n}\right) & =-\int_{0}^{T_{n}}\left\langle J^{\prime}\left(\eta\left(t, u_{n}\right)\right), \varrho\left(\eta\left(t, u_{n}\right)\right) \zeta\left(\eta\left(t, u_{n}\right)\right)\right\rangle d t \\
& =-\int_{0}^{T_{n}}\left\langle J^{\prime}\left(\eta\left(t, u_{n}\right)\right), \zeta\left(\eta\left(t, u_{n}\right)\right)\right\rangle d t \leq-a_{r} T_{n} \rightarrow-\infty .
\end{aligned}
$$

This contradicts the assumption that $J$ is bounded from below.

By (3.27) and (3.26), we see that, when $n$ is large enough, there exist $t_{n}<t_{n}^{\prime}<$ $t_{n}^{\prime \prime} \leq T_{n}$ such that

$$
\eta\left(t_{n}^{\prime}, u_{n}\right) \in c l_{w}\left(\left(\mathcal{K}^{\prime}\right)_{\iota / 4}\right), \quad \eta\left(t_{n}^{\prime \prime}, u_{n}\right) \in J^{c_{r}} \backslash\left(\mathcal{K}^{\prime}\right)_{\iota / 3},
$$

and

$$
\eta\left(\left[t_{n}^{\prime}, t_{n}^{\prime \prime}\right], u_{n}\right) \subset J^{c_{r}} \cap\left(c l_{w}\left(\left(\mathcal{K}^{\prime}\right)_{\iota / 3}\right) \backslash\left(\mathcal{K}^{\prime}\right)_{\iota / 4}\right) .
$$

Copyright (c) by SIAM. Unauthorized reproduction of this article is prohibited. 
It follows that

$$
\begin{aligned}
\frac{\iota}{12}=\frac{\iota}{3}-\frac{\iota}{4} & \leq\left\|\eta\left(t_{n}^{\prime \prime}, u_{n}\right)-\eta\left(t_{n}^{\prime}, u_{n}\right)\right\|_{w} \\
& =\left\|-\int_{t_{n}^{\prime}}^{t_{n}^{\prime \prime}} \varrho\left(\eta\left(t, u_{n}\right)\right) \zeta\left(\eta\left(t, u_{n}\right)\right) d t\right\|_{w} \\
& =\left\|\int_{t_{n}^{\prime}}^{t_{n}^{\prime \prime}} \zeta\left(\eta\left(t, u_{n}\right)\right) d t\right\|_{w} \quad(\text { by }(3.30)) \\
& \leq \int_{t_{n}^{\prime}}^{t_{n}^{\prime \prime}}\left\|\zeta\left(\eta\left(t, u_{n}\right)\right)\right\|_{w} d t \leq \int_{t_{n}^{\prime}}^{t_{n}^{\prime \prime}}\left\|\zeta\left(\eta\left(t, u_{n}\right)\right)\right\| d t
\end{aligned}
$$

By $\|\zeta\| \leq 1$ (see Lemma 3.4), we have, as a consequence of (3.31),

$$
t_{n}^{\prime \prime}-t_{n}^{\prime} \geq \frac{\iota}{12}
$$

By the conclusion (iv) in Lemma 3.4, we have

$$
b:=\inf \left\{\left\langle J^{\prime}(u), \zeta(u)\right\rangle \mid J(u)<0, u \in c l_{w}\left(\left(\mathcal{K}^{\prime}\right)_{\iota / 3} \backslash\left(\mathcal{K}^{\prime}\right)_{\iota / 4}\right)\right\}>0 .
$$

Clearly, $b$ is independent of $r$. Note that

$$
\begin{aligned}
J\left(\eta\left(t_{n}^{\prime \prime}, u_{n}\right)\right) & =J\left(\eta\left(t_{n}^{\prime}, u_{n}\right)\right)-\int_{t_{n}^{\prime}}^{t_{n}^{\prime \prime}}\left\langle J^{\prime}\left(\eta\left(t, u_{n}\right)\right), \varrho\left(\eta\left(t, u_{n}\right)\right) \zeta\left(\eta\left(t, u_{n}\right)\right)\right\rangle d t \\
& =J\left(\eta\left(t_{n}^{\prime}, u_{n}\right)\right)-\int_{t_{n}^{\prime}}^{t_{n}^{\prime \prime}}\left\langle J^{\prime}\left(\eta\left(t, u_{n}\right)\right), \zeta\left(\eta\left(t, u_{n}\right)\right)\right\rangle d t .
\end{aligned}
$$

By (3.30) and (3.32)-(3.34), we see that

$$
J\left(\eta\left(T_{n}, u_{n}\right)\right) \leq J\left(\eta\left(t_{n}^{\prime \prime}, u_{n}\right)\right) \leq J\left(\eta\left(t_{n}^{\prime}, u_{n}\right)\right)-\frac{b \iota}{12} \leq-\frac{b \iota}{12}:=c_{*},
$$

where $c_{*}$ is a constant independent of $r$. Since $J^{c_{*}}$ is $w$-compact (see Lemma 3.1) and $0 \notin J^{c_{*}}$, there exists a constant $r_{*}>0$ which is independent of $r$ such that

$$
J^{c_{*}} \cap B_{X_{w}}\left(0, r_{*}\right)=\emptyset .
$$

Choosing $0<r<r_{*}$ and using (3.26), (3.35), and (3.36), we arrive at a contradiction. This finishes the proof.

\section{Proof of Theorem 1.1.}

Proof of Theorem 1.1. Arguing indirectly, assume that there exists $\iota>0$ such that (3.5) holds. Let $r_{0}>0$ be the constant given in Lemma 3.5. By Lemma 3.1,

$$
B:=J^{0} \backslash B_{X_{w}}\left(0, r_{0}\right)
$$

is a $w$-compact set, and by the condition that $J$ is an even functional (see $\left(\mathbf{A}_{\mathbf{1}}\right)$ ), we have $B \in S\left(X_{w}\right)$. Then, by (2.3) and the property (iii) in Proposition 2.5, we have

$$
\gamma(B) \leq \gamma_{w}(B)<+\infty .
$$

Copyright (c) by SIAM. Unauthorized reproduction of this article is prohibited. 
Therefore, we can choose an integer $m>\gamma(B)$. And by the condition $\left(\mathbf{A}_{\mathbf{5}}\right)$, we can choose $A \in S(X)$ such that $\gamma(A) \geq m$ and

$$
c:=\sup _{u \in A} J(u)<0 .
$$

Then, by Lemma 3.5,

$$
\eta\left(T_{c}, A\right) \subset B .
$$

Since $\eta$ is equivariant (see (3.25)) and continuous, by the properties of the genus, we get that

$$
m \leq \gamma(A) \leq \gamma\left(\eta\left(T_{c}, A\right)\right) \leq \gamma(B)<m .
$$

This contradiction finishes the proof.

5. Application 1: Degenerate quasi-linear elliptic equations. In this section, we consider the following quasi-linear elliptic equation:

$(5.1)-\operatorname{div}\left(D_{\xi} \Psi(x, u, \nabla u)\right)+D_{s} \Psi(x, u, \nabla u)=K(x)|u|^{q-2} u \quad$ in $\Omega, \quad u=0 \quad$ on $\partial \Omega$, where $\Omega$ is a smooth bounded domain in $\mathbb{R}^{N}(N \geq 2)$.

The function

$$
\Psi: \Omega \times \mathbb{R} \times \mathbb{R}^{N} \rightarrow \mathbb{R}, \quad(x, s, \xi) \mapsto \Psi(x, s, \xi)
$$

satisfies the following conditions.

$\left(\boldsymbol{\Psi}_{\mathbf{1}}\right) \Psi: \Omega \times \mathbb{R} \times \mathbb{R}^{N} \rightarrow \mathbb{R}$ is measurable in $x$ for all $(s, \xi) \in \mathbb{R} \times \mathbb{R}^{N}$, of class $C^{1}$ in $(s, \xi)$ for almost every $x \in \Omega$. For almost every $x \in \Omega$ and $s \in \mathbb{R}$, the function $\Psi(x, s, \cdot)$ is convex.

$\left(\mathbf{\Psi}_{\mathbf{2}}\right)$ For any $(x, s, \xi) \in \Omega \times \mathbb{R} \times \mathbb{R}^{N}, \Psi(x, s, \xi) \geq 0, s D_{s} \Psi(x, s, \xi) \geq 0$, and $\Psi(x,-s,-\xi)=\Psi(x, s, \xi)$. For almost every $(x, s), \Psi(x, s, 0)=0$. And for almost every $(x, \xi), D_{s} \Psi(x, 0, \xi)=0$. Moreover, for $s \neq 0$, the function

$$
s D_{s} \Psi(x, s, \xi) \text { is convex in } \xi \text {. }
$$

$\left(\mathbf{\Psi}_{\mathbf{3}}\right)$ There exist $1<p<N, \alpha>0$, and $\lambda>0$ such that

$$
\Psi(x, s, \xi) \geq \lambda|\xi|^{p}
$$

for $(x, s) \in \Omega \times \mathbb{R}$ and $|\xi| \geq \alpha$.

$\left(\mathbf{\Psi}_{4}\right)$ There exist $L>0, C>0$, and $M>0$ such that

$$
\begin{gathered}
\left|D_{\xi} \Psi(x, s, \xi)\right| \leq L|\xi|^{p-1} \quad \forall(x, s, \xi) \in \Omega \times \mathbb{R} \times \mathbb{R}^{N}, \\
M D_{\xi} \Psi(x, s, \xi) \cdot \xi \pm D_{s} \Psi(x, s, \xi) \geq-C \quad \forall(x, s, \xi) \in \Omega \times \mathbb{R} \times \mathbb{R}^{N},
\end{gathered}
$$

and for $x \in \Omega$ a.e. and all $s \in \mathbb{R}$, the functions

$$
M D_{\xi} \Psi(x, s, \xi) \cdot \xi \pm D_{s} \Psi(x, s, \xi) \text { are convex in } \xi .
$$

Remark 5.1.

(i) Under the assumptions $\left(\boldsymbol{\Psi}_{\mathbf{1}}\right)-\left(\mathbf{\Psi}_{\mathbf{4}}\right)$, the function $\Psi(x, s, \xi)$ may not be strictly convex in $\xi$. This case has been considered by several authors in some special situations (see, for example, [11] and [22]).

Copyright (C) by SIAM. Unauthorized reproduction of this article is prohibited. 
(ii) Under the assumptions $\left(\boldsymbol{\Psi}_{\mathbf{1}}\right)-\left(\boldsymbol{\Psi}_{\mathbf{4}}\right)$, the quasi-linear elliptic operator

$$
-\operatorname{div}\left(D_{\xi} \Psi(x, u, \nabla u)\right)+D_{s} \Psi(x, u, \nabla u)
$$

may be very degenerate. An example of such a $\Psi$ is the following:

$$
\Psi(x, s, \xi)=\left(1+(\arctan s)^{2}\right) g(|\xi|),
$$

where

$$
g(r)= \begin{cases}0, & 0 \leq r \leq 2 \\ 2-\sqrt{4-(r-2)^{2}}, & 2 \leq r \leq 3 \\ a r^{p}+b r+c, & r \geq 3\end{cases}
$$

and

$$
a=\frac{4 \times 3^{1 / 2-p}}{p(p-1)}, \quad b=\frac{p-5}{\sqrt{3}(p-1)}, \quad c=2-\frac{2 \sqrt{3}(p-2)}{p} .
$$

Elementary calculations show that this $\Psi$ satisfies the assumptions $\left(\boldsymbol{\Psi}_{\mathbf{1}}\right)-\left(\boldsymbol{\Psi}_{\mathbf{4}}\right)$. Equation (5.1) with $\Psi(x, s, \cdot)$ very degenerate and not strictly convex has never been studied before, as far as we know.

(iii) Equations (5.4) and (5.5) imply that

$$
\left|D_{s} \Psi(x, s, \xi)\right| \leq M L|\xi|^{p}+C \quad \forall(x, s, \xi) \in \Omega \times \mathbb{R} \times \mathbb{R}^{N} .
$$

For the nonlinear term on the right side of (5.1), we assume

$$
1<q<p
$$

and the following assumptions.

$\left(\mathbf{K}_{\mathbf{1}}\right) K \in L^{\infty}(\Omega)$.

$\left(\mathbf{K}_{\mathbf{2}}\right)$ The following holds:

$$
\operatorname{essinf}_{x \in \Omega} K(x)>0 .
$$

From (5.4) and $\Psi(x, s, 0) \equiv 0\left(\right.$ see $\left.\left(\mathbf{\Psi}_{\mathbf{2}}\right)\right)$, we deduce that

$$
\Psi(x, s, \xi) \leq \frac{L}{p}|\xi|^{p} \quad \forall(x, s, \xi) \in \Omega \times \mathbb{R} \times \mathbb{R}^{N} .
$$

Under the assumptions $\left(\mathbf{\Psi}_{\mathbf{1}}\right)-\left(\mathbf{\Psi}_{\mathbf{4}}\right),(5.9)$, and $\left(\mathbf{K}_{\mathbf{1}}\right)-\left(\mathbf{K}_{\mathbf{2}}\right)$, since we have (5.11), the following functional is well defined:

$$
J(u)=\int_{\Omega} \Psi(x, u, \nabla u) d x-\frac{1}{q} \int_{\Omega} K(x)|u|^{q} d x, \quad u \in X:=W_{0}^{1, p}(\Omega),
$$

where $W_{0}^{1, p}(\Omega)$ is the Sobolev space with the standard norm

$$
\|u\|=\left(\int_{\Omega}|\nabla u|^{p} d x\right)^{1 / p} .
$$

By (5.4), (5.8), (5.9), and $\left(\mathbf{K}_{\mathbf{1}}\right)-\left(\mathbf{K}_{\mathbf{2}}\right)$, it is easy to see that for

$$
E:=W_{0}^{1, p}(\Omega) \cap L^{\infty}(\Omega),
$$

Copyright $@$ by SIAM. Unauthorized reproduction of this article is prohibited. 
$J$ is $E$-differentiable, and for any $u \in X$ and $h \in E$,

$$
\left\langle J^{\prime}(u), h\right\rangle=\int_{\Omega} D_{\xi} \Psi(x, u, \nabla u) \cdot \nabla h d x+\int_{\Omega} D_{s} \Psi(x, u, \nabla u) h d x-\int_{\Omega} K(x)|u|^{q-2} u h d x .
$$

It follows that the critical points of $J$ are weak solutions of (5.1).

In general, this functional does not satisfy the Palais-Smale condition. For example, in the case of (5.7), we can choose a sequence $\left\{u_{n}\right\} \subset X$ satisfying that $\sup _{x \in \Omega}\left|\nabla u_{n}\right| \leq 1$ for all $n, u_{n} \rightarrow 0$ in $X$ and $\inf _{n}\left\|u_{n}\right\|>0$. Then $\left\{u_{n}\right\}$ is a PalaisSmale sequence of $J$, but it does not contain a convergent subsequence.

Such a sequence can be constructed as follows. Denote

$$
I=\left\{x \in \mathbb{R}^{N} \mid x=\left(x_{1}, x_{2}, \ldots, x_{N}\right), 0 \leq x_{j} \leq 1\right\}
$$

and

$$
s_{j i}=\left\{x \in I \mid x_{j}=i\right\}, \quad I_{j i}=\operatorname{conv}\left(s_{j i} \cup\{(1 / 2,1 / 2, \ldots, 1 / 2)\}\right)
$$

for $j=1,2, \ldots, N$ and $i=0,1$, where $\operatorname{conv}(A)$ means the closed convex hull of a set $A$. Then $I=\cup_{0 \leq j \leq 1, i=0,1} I_{j i}$. Define, for $x \in I$,

$$
v(x)= \begin{cases}x_{j} & \text { if } x \in I_{j 0} \\ 1-x_{j} & \text { if } x \in I_{j 1}\end{cases}
$$

Then $v$ is well defined, $v \in C_{0}(I)$, and $|\nabla v|=1$ a.e. in $I$. Now suppose, without loss of generality, that we can translate $I$ into a new cube $I^{*}$ which is contained in $\Omega$. For any $n$, divide $I^{*}$ equally into $n^{N}$ small cubes. Define, for $x \in \frac{1}{n} I:=\{x \mid n x \in I\}$,

$$
v_{n}(x)=\frac{1}{n} v(n x) .
$$

Define $u_{n}$ on $I^{*}$ in such a way that its constraint on each small cube is a translation of $v_{n}$. Outside of $I^{*}, u_{n}$ is defined to be 0 . For such a sequence, we have $\left\{u_{n}\right\} \subset X$, $\left|\nabla u_{n}\right|=1$ a.e. in $I^{*}$, and $\left|\nabla u_{n}\right|=0$ in $\Omega \backslash I^{*}$ for all $n$, and therefore $\left\|u_{n}\right\|=1$ for all $n$. Moreover, $\left\|u_{n}\right\|_{L^{\infty}(\Omega)} \rightarrow 0$ and thus $u_{n} \rightarrow 0$ in $X$ as $n \rightarrow \infty$.

The main result of this section is the following theorem.

ThEOREM 5.2. Under the assumptions $\left(\mathbf{\Psi}_{\mathbf{1}}\right)-\left(\mathbf{\Psi}_{\mathbf{4}}\right),(5.9)$, and $\left(\mathbf{K}_{\mathbf{1}}\right)-\left(\mathbf{K}_{\mathbf{2}}\right),(5.1)$ has infinitely many solutions.

Proof. We shall verify that the functional $J$ defined in (5.12) satisfies the assumptions $\left(\mathbf{A}_{\mathbf{1}}\right)-\left(\mathbf{A}_{\mathbf{5}}\right)$ in Theorem 1.1 .

Since $1<q<p$, by the Sobolev inequality and $\left(\mathbf{K}_{\mathbf{1}}\right)$, there exists $C>0$ such that

$$
\int_{\Omega} K(x)|u|^{q} d x \leq C\|u\|^{q} \quad \forall u \in X .
$$

By $\left(\mathbf{\Psi}_{\mathbf{3}}\right),(5.11)$, and $(5.14)$, we have

$$
\begin{aligned}
J(u) & =\int_{|\nabla u| \geq \alpha} \Psi(x, u, \nabla u) d x+\int_{|\nabla u|<\alpha} \Psi(x, u, \nabla u) d x-\frac{1}{q} \int_{\Omega} K(x)|u|^{q} d x \\
& \geq \lambda \int_{|\nabla u| \geq \alpha}|\nabla u|^{p} d x-\frac{L}{p} \int_{|\nabla u|<\alpha}|\nabla u|^{p} d x-\frac{C}{q}\|u\|^{q} \\
& \geq \lambda\|u\|^{p}-\frac{C}{q}\|u\|^{q}-\left(\frac{L}{p}+\lambda\right) \alpha^{p} \operatorname{meas}(\Omega),
\end{aligned}
$$

Copyright (c) by SIAM. Unauthorized reproduction of this article is prohibited. 
where meas $(A)$ denotes the Lebesgue measure of a set $A$. Therefore, $J$ is coercive and bounded from below. It follows that $\{u \in X \mid J(u) \leq 0\}$ is bounded in $X$.

By $\left(\mathbf{\Psi}_{\mathbf{1}}\right)$ and $\left(\mathbf{\Psi}_{\mathbf{2}}\right)$, we see that $\int_{\Omega} \Psi(x, u, \nabla u) d x$ is weakly sequentially lower semicontinuous (see [25, Theorem 1.6]). Since the embedding $X \hookrightarrow L^{q}(\Omega)$ is compact (see, for example, [1]), the functional $\int_{\Omega} K(x)|u|^{q} d x$ is weakly sequentially continuous. Therefore, $J$ is weakly sequentially lower semicontinuous. We have verified that $J$ satisfies the assumptions $\left(\mathbf{A}_{\mathbf{1}}\right),\left(\mathbf{A}_{\mathbf{3}}\right)$, and $\left(\mathbf{A}_{\mathbf{4}}\right)$ in Theorem 1.1.

Now, we prove that $J$ satisfies the assumption $\left(\mathbf{A}_{\mathbf{2}}\right)$ in Theorem 1.1. Let $u \in X$ and $\left\{u_{n}\right\} \subset X$ satisfy that $\left|J^{\prime}\left(u_{n}\right)\right| \rightarrow 0$ and $u_{n} \rightarrow u$ as $n \rightarrow \infty$. Choose $H \in C^{1}(\mathbb{R})$ such that $H(s)=1$ for $|s| \leq 1 / 2, H(s)=0$ for $|s| \geq 1,0 \leq H(s) \leq 1$, and $\left|H^{\prime}(s)\right| \leq 4$ for all s. Let $\psi \in E$ with $\psi \geq 0$ and let $T \in \mathbb{R}$ with $T \geq 1$. As in [4], take $\varphi_{n}=\psi H\left(u_{n} / T\right) \exp \left(-M u_{n}^{+}\right)$as a test function in $\left\langle J^{\prime}\left(u_{n}\right), \varphi_{n}\right\rangle=o(1)$, where $M>0$ is the constant in (5.5) and $u_{n}^{+}=\max \left\{u_{n}, 0\right\}$. By (5.13), we have

$$
\begin{aligned}
o(1)= & -\int_{\Omega}\left(M D_{\xi} \Psi\left(x, u_{n}, \nabla u_{n}\right) \cdot \nabla u_{n}^{+}-D_{s} \Psi\left(x, u_{n}, \nabla u_{n}\right)\right) \psi H\left(u_{n} / T\right) \exp \left(-M u_{n}^{+}\right) d x \\
& +\int_{\Omega} \exp \left(-M u_{n}^{+}\right) D_{\xi} \Psi\left(x, u_{n}, \nabla u_{n}\right) \cdot \nabla\left(\psi H\left(u_{n} / T\right)\right) d x \\
& -\int_{\Omega} K(x)\left|u_{n}\right|^{q-2} u_{n} \psi H\left(u_{n} / T\right) \exp \left(-M u_{n}^{+}\right) d x .
\end{aligned}
$$

Letting $\Omega_{n}^{ \pm}:=\left\{x \in \Omega \mid \pm u_{n}(x) \geq 0\right\}$, we rewrite (5.16) as

$$
\begin{aligned}
o(1)= & -\int_{\Omega_{n}^{+}}\left(M D_{\xi} \Psi\left(x, u_{n}, \nabla u_{n}\right) \cdot \nabla u_{n}-D_{s} \Psi\left(x, u_{n}, \nabla u_{n}\right)\right) \psi H\left(u_{n} / T\right) \exp \left(-M u_{n}^{+}\right) d x \\
& +\int_{\Omega_{n}^{-}} D_{s} \Psi\left(x, u_{n}, \nabla u_{n}\right) \psi H\left(u_{n} / T\right) \exp \left(-M u_{n}^{+}\right) d x \\
& +\int_{\Omega} \exp \left(-M u_{n}^{+}\right) D_{\xi} \Psi\left(x, u_{n}, \nabla u_{n}\right) \cdot \nabla\left(\psi H\left(u_{n} / T\right)\right) d x \\
& -\int_{\Omega} K(x)\left|u_{n}\right|^{q-2} u_{n} \psi H\left(u_{n} / T\right) \exp \left(-M u_{n}^{+}\right) d x .
\end{aligned}
$$

Since $u_{n} \rightarrow u$ in $X$, we have, up to a subsequence,

$$
u_{n} \rightarrow u \text { a.e. in } \Omega \text { and } u_{n} \rightarrow u \text { in } L^{t}(\Omega) \text { for all } t \in[1, N p /(N-p)) .
$$

By (5.5) and (5.6), we may apply [25, Theorem 1.6] to the function

$$
\left(M D_{\xi} \Psi(x, s, \xi) \cdot \xi-D_{s} \Psi(x, s, \xi)\right) \psi H(s / T) \exp \left(-M s \chi_{+}(s)\right) \chi_{+}(s),
$$

where $\chi_{+}(s)=1$ if $s>0$ and $\chi_{+}(s)=0$ if $s \leq 0$, to obtain that

$$
\begin{aligned}
& \liminf _{n \rightarrow \infty} \int_{\Omega_{n}^{+}}\left(M D_{\xi} \Psi\left(x, u_{n}, \nabla u_{n}\right) \cdot \nabla u_{n}-D_{s} \Psi\left(x, u_{n}, \nabla u_{n}\right)\right) \psi H\left(u_{n} / T\right) \exp \left(-M u_{n}^{+}\right) d x \\
\geq & \int_{\Omega^{+}}\left(M D_{\xi} \Psi(x, u, \nabla u) \cdot \nabla u-D_{s} \Psi(x, u, \nabla u)\right) \psi H(u / T) \exp \left(-M u^{+}\right) d x,
\end{aligned}
$$

Copyright (c) by SIAM. Unauthorized reproduction of this article is prohibited. 
where $\Omega^{+}=\{x \in \Omega \mid u(x) \geq 0\}$ and $u^{+}=\max \{u, 0\}$. According to $\left(\mathbf{\Psi}_{\mathbf{2}}\right)$, we have $D_{s} \Psi(x, s, \xi) \leq 0$, and $-D_{s} \Psi(x, s, \xi)$ is convex in $\xi$ if $s \leq 0$. Using [25, Theorem 1.6] again, we have

$$
\begin{aligned}
& \quad \limsup _{n \rightarrow \infty} \int_{\Omega_{n}^{-}} D_{s} \Psi\left(x, u_{n}, \nabla u_{n}\right) \psi H\left(u_{n} / T\right) \exp \left(-M u_{n}^{+}\right) d x \\
\leq & \int_{\Omega^{-}} D_{s} \Psi(x, u, \nabla u) \psi H(u / T) \exp \left(-M u^{+}\right) d x,
\end{aligned}
$$

where $\Omega^{-}=\{x \in \Omega \mid u(x) \leq 0\}$.

In order to study the third term on the right side of (5.17), we use an argument from the proof of [2, Theorem 2.1] (see also [10]) to verify that, up to a subsequence,

$$
e_{n}:=\left(D_{\xi} \Psi\left(x, u_{n}, \nabla u_{n}\right)-D_{\xi} \Psi(x, u, \nabla u)\right) \cdot\left(\nabla u_{n}-\nabla u\right) \rightarrow 0 \text { a.e. in } \Omega .
$$

The convexity of $\Psi$ in $\xi$ implies that $e_{n} \geq 0$ in $\Omega$, while (5.4), together with the fact that $\left\{u_{n}\right\}$ is bounded in $X$, implies that $\left\{e_{n}\right\}$ is bounded in $L^{1}(\Omega)$. For $\eta>0$, define $T_{\eta}(s)=s$ for $|s| \leq \eta$ and $T_{\eta}(s)=(s /|s|) \eta$ for $|s|>\eta$. Using $\varphi_{n}=T_{\eta}\left(u_{n}-u\right)$ as a test function in $\left\langle J^{\prime}\left(u_{n}\right), \varphi_{n}\right\rangle=o(1)$, we have

$$
\begin{aligned}
o(1)= & \int_{\Omega}\left(D_{\xi} \Psi\left(x, u_{n}, \nabla u_{n}\right)-D_{\xi} \Psi(x, u, \nabla u)\right) \cdot \nabla\left(T_{\eta}\left(u_{n}-u\right)\right) d x \\
& +\int_{\Omega} D_{s} \Psi\left(x, u_{n}, \nabla u_{n}\right) T_{\eta}\left(u_{n}-u\right) d x \\
& +\int_{\Omega} D_{\xi} \Psi(x, u, \nabla u) \cdot \nabla\left(T_{\eta}\left(u_{n}-u\right)\right) d x \\
& -\int_{\Omega} K(x)\left|u_{n}\right|^{q-2} u_{n} T_{\eta}\left(u_{n}-u\right) d x
\end{aligned}
$$

For any $\eta>0$, by (5.4), $\left(\mathbf{K}_{\mathbf{1}}\right)$, and (5.18), since $\nabla\left(T_{\eta}\left(u_{n}-u\right)\right) \rightarrow 0$ weakly in $L^{p}(\Omega)$ and $T_{\eta}\left(u_{n}-u\right) \rightarrow 0$ strongly in $L^{t}(\Omega)$ for all $t \in[1, N p /(N-p))$, the last two terms in (5.22) converge to 0 . As a consequence of (5.8), the second term on the right side of $(5.22)$ is estimated as

$$
\int_{\Omega}\left|D_{s} \Psi\left(x, u_{n}, \nabla u_{n}\right) T_{\eta}\left(u_{n}-u\right)\right| d x \leq C \eta .
$$

Therefore, (5.22) implies

$$
\limsup _{n \rightarrow \infty} \int_{\left\{\left|u_{n}-u\right| \leq \eta\right\}} e_{n} d x \leq C \eta .
$$

Fix a number $\theta \in(0,1)$. Using the Hölder inequality, we see that

$$
\begin{aligned}
\int_{\Omega} e_{n}^{\theta} d x \leq & \left(\int_{\left\{\left|u_{n}-u\right| \leq \eta\right\}} e_{n} d x\right)^{\theta}(\operatorname{meas}(\Omega))^{1-\theta} \\
& +\left(\int_{\Omega} e_{n} d x\right)^{\theta}\left(\operatorname{meas}\left(\left\{\left|u_{n}-u\right| \geq \eta\right\}\right)\right)^{1-\theta} .
\end{aligned}
$$

Since $\left\{e_{n}\right\}$ is bounded in $L^{1}(\Omega)$ and $u_{n}$ converges to $u$ in measure, combining (5.23) and (5.24) yields

$$
\limsup _{n \rightarrow \infty} \int_{\Omega} e_{n}^{\theta} d x \leq C \eta^{\theta}
$$

Copyright $\odot$ by SIAM. Unauthorized reproduction of this article is prohibited. 
for any $\eta>0$. Since $\eta>0$ is arbitrary, one has

$$
\lim _{n \rightarrow \infty} \int_{\Omega} e_{n}^{\theta} d x=0
$$

which implies (5.21) up to a subsequence. From (5.18) and (5.21), we use Lemma 7.1 to deduce that

$$
D_{\xi} \Psi\left(x, u_{n}, \nabla u_{n}\right) \rightarrow D_{\xi} \Psi(x, u, \nabla u) \text { a.e. in } \Omega .
$$

By (5.4), we have

$$
\sup _{n} \int_{\Omega}\left|\exp \left(-M u_{n}^{+}\right) H\left(u_{n} / T\right) D_{\xi} \Psi\left(x, u_{n}, \nabla u_{n}\right)\right|^{\frac{p}{p-1}} d x<+\infty,
$$

which combined with (5.25) implies

$$
\begin{aligned}
& \exp \left(-M u_{n}^{+}\right) H\left(u_{n} / T\right) D_{\xi} \Psi\left(x, u_{n}, \nabla u_{n}\right) \rightarrow \\
& \exp \left(-M u^{+}\right) H(u / T) D_{\xi} \Psi(x, u, \nabla u) \quad \text { in } L^{\frac{p}{p-1}}(\Omega) .
\end{aligned}
$$

Therefore,

$$
\begin{aligned}
& \lim _{n \rightarrow \infty} \int_{\Omega} \exp \left(-M u_{n}^{+}\right) H\left(u_{n} / T\right) D_{\xi} \Psi\left(x, u_{n}, \nabla u_{n}\right) \cdot \nabla \psi d x \\
= & \int_{\Omega} \exp \left(-M u^{+}\right) H(u / T) D_{\xi} \Psi(x, u, \nabla u) \cdot \nabla \psi d x .
\end{aligned}
$$

Denoting

$$
D_{0}=\sup _{n}\left\|u_{n}\right\|^{p}, \quad \mu=\|\psi\|_{L^{\infty}(\Omega)},
$$

by (5.4) and the fact that $\left|H^{\prime}(s)\right| \leq 4$ for all $s$, we have

$$
\limsup _{n \rightarrow \infty} \frac{1}{T} \int_{\Omega}\left|\psi \exp \left(-M u_{n}^{+}\right) H^{\prime}\left(u_{n} / T\right) D_{\xi} \Psi\left(x, u_{n}, \nabla u_{n}\right) \cdot \nabla u_{n}\right| d x \leq \frac{4 \mu L D_{0}}{T}
$$

and

$$
\frac{1}{T} \int_{\Omega}\left|\psi \exp \left(-M u^{+}\right) H^{\prime}(u / T) D_{\xi} \Psi(x, u, \nabla u) \cdot \nabla u\right| d x \leq \frac{4 \mu L D_{0}}{T} .
$$

Combining (5.27), (5.28), and (5.29) leads to

$$
\begin{aligned}
\limsup _{n \rightarrow \infty} & \mid \int_{\Omega} \exp \left(-M u_{n}^{+}\right) D_{\xi} \Psi\left(x, u_{n}, \nabla u_{n}\right) \cdot \nabla\left(\psi H\left(u_{n} / T\right)\right) d x \\
& -\int_{\Omega} \exp \left(-M u^{+}\right) D_{\xi} \Psi(x, u, \nabla u) \cdot \nabla(\psi H(u / T)) d x \mid \leq \frac{8 \mu L D_{0}}{T} .
\end{aligned}
$$

By (5.18), we have

$$
\begin{aligned}
& \lim _{n \rightarrow \infty} \int_{\Omega} K(x)\left|u_{n}\right|^{q-2} u_{n} \psi H\left(u_{n} / T\right) \exp \left(-M u_{n}^{+}\right) d x \\
= & \int_{\Omega} K(x)|u|^{q-2} u \psi H(u / T) \exp \left(-M u^{+}\right) d x .
\end{aligned}
$$

Copyright (c) by SIAM. Unauthorized reproduction of this article is prohibited. 
Combining (5.17), (5.19), (5.20), (5.30), and (5.31) yields

$$
\begin{aligned}
& \int_{\Omega} D_{\xi} \Psi(x, u, \nabla u) \nabla\left(\psi H(u / T) \exp \left(-M u^{+}\right)\right) d x \\
& +\int_{\Omega} D_{s} \Psi(x, u, \nabla u) \psi H(u / T) \exp \left(-M u^{+}\right) d x \\
& -\int_{\Omega} K(x)|u|^{q-2} u \psi H(u / T) \exp \left(-M u^{+}\right) d x \geq-8 \mu L D_{0} / T
\end{aligned}
$$

Letting $T \rightarrow+\infty$ in (5.32), we obtain

$$
\begin{aligned}
& \int_{\Omega} D_{\xi} \Psi(x, u, \nabla u) \nabla\left(\psi \exp \left(-M u^{+}\right)\right) d x+\int_{\Omega} D_{s} \Psi(x, u, \nabla u) \psi \exp \left(-M u^{+}\right) d x \\
& -\int_{\Omega} K(x)|u|^{q-2} u \psi \exp \left(-M u^{+}\right) d x \geq 0 .
\end{aligned}
$$

Let $\varphi \in E$ with $\varphi \geq 0$. For $n \in \mathbb{N}$, define

$$
u^{+n}(x)= \begin{cases}0, & u(x) \leq 0 \\ u(x), & 0<u(x) \leq n \\ n, & u(x)>n\end{cases}
$$

and take $\psi=\varphi \exp \left(M u^{+n}\right)$ in (5.33). It follows that

$$
\begin{aligned}
& \int_{\Omega} D_{\xi} \Psi(x, u, \nabla u) \nabla\left(\varphi \exp \left(-M\left(u^{+}-u^{+n}\right)\right)\right) d x \\
& +\int_{\Omega} D_{s} \Psi(x, u, \nabla u) \varphi \exp \left(-M\left(u^{+}-u^{+n}\right)\right) d x \\
& -\int_{\Omega} K(x)|u|^{q-2} u \varphi \exp \left(-M\left(u^{+}-u^{+n}\right)\right) d x \geq 0 .
\end{aligned}
$$

Letting $n \rightarrow+\infty$ in (5.34), we deduce that, for any $\varphi \in E$ with $\varphi \geq 0$,

$$
\int_{\Omega} D_{\xi} \Psi(x, u, \nabla u) \nabla \varphi d x+\int_{\Omega} D_{s} \Psi(x, u, \nabla u) \varphi d x-\int_{\Omega} K(x)|u|^{q-2} u \varphi d x \geq 0 .
$$

Repeating the argument above with test function $\varphi_{n}=\psi H\left(u_{n} / T\right) \exp \left(-M u_{n}^{-}\right)$, where $u_{n}^{-}=u_{n}^{+}-u_{n}$, we obtain, for any $\varphi \in E$ with $\varphi \geq 0$,

$$
\int_{\Omega} D_{\xi} \Psi(x, u, \nabla u) \nabla \varphi d x+\int_{\Omega} D_{s} \Psi(x, u, \nabla u) \varphi d x-\int_{\Omega} K(x)|u|^{q-2} u \varphi d x \leq 0 .
$$

By (5.35) and (5.36), we have $\left|J^{\prime}(u)\right|=0$. Therefore, ( $\left.\mathbf{A}_{\mathbf{2}}\right)$ holds for $J$.

Finally, by (5.11), we have

$$
J(u) \leq \frac{L}{p}\|u\|^{p}-\frac{1}{q} \int_{\Omega} K(x)|u|^{q} d x, \quad u \in X .
$$

Since $1<q<p$, by (5.10), for any $k \in \mathbb{N}$, if $X_{k}$ is a $k$-dimensional subspace of $C_{0}^{\infty}(\Omega)$ and $\rho_{k}>0$ is sufficiently small, then $\sup _{X_{k} \cap S_{\rho}} J<0$, where $S_{\rho}=\{u \in X \mid\|u\|=\rho\}$. Therefore, $J$ satisfies $\left(\mathbf{A}_{\mathbf{1}}\right)-\left(\mathbf{A}_{\mathbf{5}}\right)$ in Theorem 1.1. By Theorem 1.1, $J$ has infinitely many critical points $\left\{u_{k}\right\}$ satisfying $u_{k} \neq 0$ for all $k$ and $u_{k} \rightarrow 0$ as $k \rightarrow \infty$.

Copyright $@$ by SIAM. Unauthorized reproduction of this article is prohibited. 
Remark 5.3. Let us remark that the assumptions on $K$ in Theorem 5.2 are not the weakest assumptions. For the example of (5.7), the assumption $\left(\mathbf{K}_{\mathbf{2}}\right)$ can be weakened as $\operatorname{essinf}_{x \in \Omega} K(x) \geq 0$. In fact, if $K=0$ a.e. on a subset $\tilde{\Omega} \subset \Omega$ having positive measure, then all $u$ with $u=0$ on $\Omega \backslash \tilde{\Omega}$ and $|D u| \leq 2$ a.e. is a solution of (5.1), and in this case we have trivially a continuum of solutions, while if $\operatorname{essinf}_{x \in \Omega} K(x)>0$, then the existence of infinitely many solutions is not a trivial problem but can be derived from Theorem 5.2.

\section{Application 2: Homoclinic orbits of second-order Hamiltonian sys-} tems. In this section, we consider the second-order Hamiltonian system

$$
\ddot{u}+\nabla_{u} V(t, u)=0,
$$

where $t \in \mathbb{R}$ and the function $V: \mathbb{R} \times \mathbb{R}^{n} \rightarrow \mathbb{R}$ satisfies the following assumptions.

$\left(\mathbf{H}_{\mathbf{1}}\right) V(t, u)=-K(t, u)+W(t, u), \nabla_{u} V$ exists, and $\nabla_{u} V \in C\left(\mathbb{R} \times \mathbb{R}^{n}, \mathbb{R}^{n}\right)$.

$\left(\mathbf{H}_{2}\right)$ There are constants $\tau_{1}, \tau_{2}>0$ such that for all $(t, u) \in \mathbb{R} \times \mathbb{R}^{n}$,

$$
\tau_{1}|u|^{2} \leq K(t, u) \leq \tau_{2}|u|^{2} .
$$

$\left(\mathbf{H}_{3}\right)$ There exists $t_{0} \in \mathbb{R}$ and $\varsigma>0$ such that uniformly in $t \in\left(t_{0}-\varsigma, t_{0}+\varsigma\right)$,

$$
\lim _{|u| \rightarrow 0} \frac{W(t, u)}{|u|^{2}}=+\infty .
$$

$\left(\mathbf{H}_{\mathbf{4}}\right)$ There exists $\vartheta \in(1,2)$ such that for all $(t, u) \in \mathbb{R} \times \mathbb{R}^{n}$,

$$
|W(t, u)| \leq b(t)|u|^{\vartheta},
$$

where $b: \mathbb{R} \rightarrow \mathbb{R}^{+}$is a function such that $b \in L^{\xi}(\mathbb{R})$ for some $1 \leq \xi \leq$ $2 /(2-\vartheta)$.

$\left(\mathbf{H}_{5}\right)$ For all $(t, u) \in \mathbb{R} \times \mathbb{R}^{n}, V(t,-u)=V(t, u)$.

We say that a classical solution $u \in C^{2}\left(\mathbb{R}, \mathbb{R}^{n}\right)$ of (6.1) is a homoclinic (to 0 ) solution if $u(t) \rightarrow 0$ and $u^{\prime}(t) \rightarrow 0$ as $t \rightarrow \infty$. Moreover, if $u(t) \not \equiv 0, u(t)$ is called a nontrivial homoclinic solution.

THEOREM 6.1. Under the assumptions $\left(\mathbf{H}_{\mathbf{1}}\right)-\left(\mathbf{H}_{\mathbf{5}}\right),(6.1)$ possesses infinitely many nontrivial homoclinic solutions.

The functional corresponding to (6.1) is

$$
J(u)=\frac{1}{2} \int_{\mathbb{R}}\left|u^{\prime}\right|^{2} d t+\int_{\mathbb{R}} K(t, u) d t-\int_{\mathbb{R}} W(t, u) d t, \quad u \in X:=H^{1}(\mathbb{R}),
$$

where

$$
H^{1}(\mathbb{R})=\left\{u:\left.\mathbb{R} \rightarrow \mathbb{R}^{n}\left|\int_{\mathbb{R}}\right| u^{\prime}\right|^{2} d t+\int_{\mathbb{R}}|u|^{2} d t<+\infty\right\}
$$

is the standard Sobolev space with the inner product and the norm

$$
(u, v)=\int_{\mathbb{R}} u^{\prime} \cdot v^{\prime} d t+\int_{\mathbb{R}} u \cdot v d t, \quad\|u\|=(u, u)^{1 / 2},
$$

respectively.

Under the assumptions $\left(\mathbf{H}_{\mathbf{1}}\right)-\left(\mathbf{H}_{\mathbf{5}}\right), J$ is in $C(X)$ but is not in $C^{1}(X)$. A typical example is the following:

$$
K(t, u)=\frac{1}{2}\left(1+\frac{1}{2} \sin \left(\frac{1}{|u|^{\alpha}}\right)\right)|u|^{2}, \quad W(t, u)=b(t)|u|^{\vartheta},
$$

Copyright $@$ by SIAM. Unauthorized reproduction of this article is prohibited. 
where $0<\alpha<1,1<\vartheta<2, b \in C(\mathbb{R}, \mathbb{R}) \cap L^{\xi}(\mathbb{R})$ for some $1 \leq \xi \leq 2 /(2-\vartheta), b \geq 0$, and $b \not \equiv 0$. For $u \in X$ and $v \in H^{1}(\mathbb{R}) \cap L^{1}(\mathbb{R})$, the derivative of $J$ at $u$ in the direction of $v$ is

$$
\begin{aligned}
\left\langle J^{\prime}(u), v\right\rangle= & \int_{\mathbb{R}} u^{\prime} \cdot v^{\prime} d t+\int_{\mathbb{R}} u \cdot v d t+\frac{1}{2} \int_{\mathbb{R}} \sin \left(\frac{1}{|u|^{\alpha}}\right) u \cdot v d t \\
& -\frac{\alpha}{4} \int_{\mathbb{R}}|u|^{-\alpha} \cos \left(\frac{1}{|u|^{\alpha}}\right) u \cdot v d t-\vartheta \int_{\mathbb{R}} b(t)|u|^{\vartheta-2} u \cdot v d t .
\end{aligned}
$$

There exist $u, v \in X$ such that the integral $\int_{\mathbb{R}}|u|^{-\alpha} \cos \left(|u|^{-\alpha}\right) u \cdot v d t$ is divergent, and therefore $J$ is not a $C^{1}$ functional in $X$. Indeed, define, for $t \in \mathbb{R}$,

$$
\begin{gathered}
\kappa(t)=\frac{1}{1+|t|^{\frac{1}{2-\alpha}}}, \\
u(t)=(\kappa(t), 0, \ldots, 0), \quad v(t)=\left(\kappa(t) \cos \left(|\kappa(t)|^{-\alpha}\right), 0, \ldots, 0\right) .
\end{gathered}
$$

Then calculations show that $u, v \in X$ and $\int_{\mathbb{R}}|u|^{-\alpha} \cos \left(|u|^{-\alpha}\right) u \cdot v d t=\infty$.

To appeal to the classical Clark theorem, which is in the framework of $C^{1}$ functionals, the following assumption on $K$ is imposed by many authors in their works on (6.1) (see, for example, [27]).

$\left(\mathbf{H}_{\mathbf{2}}^{\prime}\right) K(t, u)=(L(t) u) \cdot u$, where $L \in C\left(\mathbb{R}, \mathbb{R}^{n^{2}}\right)$ is a symmetric and positive definite matrix for all $t \in \mathbb{R}$ and there are constants $0<\tau_{1}<\tau_{2}$ such that for all $(t, u) \in \mathbb{R} \times \mathbb{R}^{n}$,

$$
\tau_{1}|u|^{2} \leq(L(t) u) \cdot u \leq \tau_{2}|u|^{2} .
$$

It is easy to verify that if $V$ satisfies $\left(\mathbf{H}_{\mathbf{1}}\right),\left(\mathbf{H}_{\mathbf{2}}^{\prime}\right)$, and $\left(\mathbf{H}_{\mathbf{3}}\right)-\left(\mathbf{H}_{\mathbf{5}}\right)$, then $J$ is a $C^{1}$ functional in $X$ and satisfies the Palais-Smale condition, and the existence of infinitely many homoclinic orbits can be derived from the classical Clark theorem. However, since no growth constraints are imposed on $\nabla_{u} V$ in the setting of $\left(\mathbf{H}_{\mathbf{1}}\right)-\left(\mathbf{H}_{\mathbf{5}}\right)$, the functional $J$ defined in (6.5) is neither in $C^{1}$ nor does it satisfy the Palais-Smale condition.

Although a variant of Clark's theorem for nonsmooth functionals was recently proved in [21], the $E$-differentiable functional in [21, Theorem 3.9] still needs to satisfy the Palais-Smale condition, and therefore [21, Theorem 3.9] is not applicable here either. When $K$ satisfies only the "pinching" condition (6.2), as far as we know, there are no known results on the existence of multiple homoclinic orbits for (6.1).

Proof of Theorem 6.1. Let $E=H^{1}(\mathbb{R}) \cap C_{0}^{\infty}(\mathbb{R})$. Since $H^{1}(\mathbb{R})$ can be embedded continuously into $L^{\infty}(\mathbb{R})$, we deduce that under the assumption $\left(\mathbf{H}_{1}\right), J$ is $E$ differentiable and, for any $u \in X$ and $h \in E$,

$$
\left\langle J^{\prime}(u), h\right\rangle=\int_{\mathbb{R}} u^{\prime} \cdot h^{\prime} d x+\int_{\mathbb{R}} \nabla_{u} V(t, u) \cdot h d x .
$$

$J$ is even as a consequence of $\left(\mathbf{H}_{\mathbf{5}}\right)$. By (6.2) and (6.4), if $\xi=1$, then

$$
\begin{aligned}
J(u) & \geq \frac{1}{2} \int_{\mathbb{R}}\left|u^{\prime}\right|^{2} d t+\tau_{1} \int_{\mathbb{R}}|u|^{2} d t-\|u\|_{L^{\infty}(\mathbb{R})} \int_{\mathbb{R}}|b(t)| d t \\
& \geq \frac{1}{2} \int_{\mathbb{R}}\left|u^{\prime}\right|^{2} d t+\tau_{1} \int_{\mathbb{R}}|u|^{2} d t-\|u\|^{\vartheta} \int_{\mathbb{R}}|b(t)| d t \\
& \geq \min \left\{\frac{1}{2}, \tau_{1}\right\}\|u\|^{2}-\|u\|^{\vartheta} \int_{\mathbb{R}}|b(t)| d t,
\end{aligned}
$$

Copyright $@$ by SIAM. Unauthorized reproduction of this article is prohibited. 
where the inequality $\|u\|_{L^{\infty}(\mathbb{R})} \leq\|u\|$ has been used. If $1<\xi \leq 2 /(2-\vartheta)$, then $\vartheta \xi /(\xi-1) \geq 2$ and we have

$$
\begin{aligned}
J(u) & \geq \frac{1}{2} \int_{\mathbb{R}}\left|u^{\prime}\right|^{2} d t+\tau_{1} \int_{\mathbb{R}}|u|^{2} d t-\left(\int_{\mathbb{R}}|b(t)|^{\xi} d t\right)^{1 / \xi}\left(\int_{\mathbb{R}}|u|^{\frac{\vartheta \xi}{\xi-1}} d t\right)^{\frac{\xi-1}{\xi}} \\
& \geq \frac{1}{2} \int_{\mathbb{R}}\left|u^{\prime}\right|^{2} d t+\tau_{1} \int_{\mathbb{R}}|u|^{2} d t-\|u\|^{\vartheta}\left(\int_{\mathbb{R}}|b(t)|^{\xi} d t\right)^{1 / \xi} \\
& \geq \min \left\{\frac{1}{2}, \tau_{1}\right\}\|u\|^{2}-\|u\|^{\vartheta}\left(\int_{\mathbb{R}}|b(t)|^{\xi} d t\right)^{1 / \xi}
\end{aligned}
$$

where the inequality $\|u\|_{L^{\vartheta \xi /(\xi-1)(\mathbb{R})}} \leq\|u\|$ has been used. It follows from (6.8) and (6.9) that $J$ is coercive and bounded from below. Therefore, $J$ satisfies the conditions $\left(\mathbf{A}_{1}\right)$ and $\left(\mathbf{A}_{4}\right)$.

Let $u_{n} \rightarrow u$ in $X$ and $h \in E$. Then

$$
\int_{\mathbb{R}} u_{n}^{\prime} \cdot h^{\prime} d t+\int_{\mathbb{R}} u_{n} \cdot h d t \rightarrow \int_{\mathbb{R}} u^{\prime} \cdot h^{\prime} d t+\int_{\mathbb{R}} u \cdot h d t .
$$

Since $h \in C_{0}^{\infty}(\mathbb{R})$, by the Lebesgue convergence theorem, we have

$$
-\int_{\mathbb{R}} u_{n} \cdot h d t+\int_{\mathbb{R}} \nabla_{u} V\left(t, u_{n}\right) \cdot h d t \rightarrow-\int_{\mathbb{R}} u \cdot h d t+\int_{\mathbb{R}} \nabla_{u} V(t, u) \cdot h d t .
$$

Combining (6.10) and (6.11) yields

$$
\left\langle J^{\prime}\left(u_{n}\right), h_{n}\right\rangle \rightarrow\left\langle J^{\prime}(u), h\right\rangle .
$$

Therefore, the condition $\left(\mathbf{A}_{\mathbf{2}}^{\prime}\right)$ in Remark 1.3 holds for $J$. Moreover, if $u_{n} \rightarrow u$ in $X$, then by $[25$, Theorem 1.6] we have

$$
\liminf _{n \rightarrow \infty} \frac{1}{2} \int_{\mathbb{R}}\left|u_{n}^{\prime}\right|^{2} d t \geq \frac{1}{2} \int_{\mathbb{R}}\left|u^{\prime}\right|^{2} d t
$$

Applying Fatou's lemma and using (6.2) leads to

$$
\liminf _{n \rightarrow \infty} \frac{1}{2} \int_{\mathbb{R}} K\left(t, u_{n}\right) d t \geq \frac{1}{2} \int_{\mathbb{R}} K(t, u) d t,
$$

while (6.4) implies

$$
\lim _{n \rightarrow \infty} \int_{\mathbb{R}} W\left(t, u_{n}\right) d t=\int_{\mathbb{R}} W(t, u) d t .
$$

By (6.13)-(6.15), we see that $J$ satisfies the condition $\left(\mathbf{A}_{\mathbf{3}}\right)$.

Finally, as in the proof of [21, Theorem 3.1], by (6.3), we deduce that the functional (6.5) satisfies the condition $\left(\mathbf{A}_{\mathbf{5}}\right)$. Then by Theorem 1.1 and Remark 1.3, (6.1) has infinitely many homoclinic solutions.

7. Appendix. In this appendix, we prove the following lemma, which is an adaptation of [22, Lemma 2.1] and which is used in the proof of Theorem 5.2. 
Lemma 7.1. Let $\Phi, \Phi_{k} \in C^{1}\left(\mathbb{R}^{N}, \mathbb{R}\right)$ be convex functions satisfying that $\Phi_{k}(\zeta) \rightarrow$ $\Phi(\zeta)$ and $\nabla \Phi_{k}(\zeta) \rightarrow \nabla \Phi(\zeta)$ for any $\zeta \in \mathbb{R}^{N}$ as $k \rightarrow \infty$, and that there exists $\gamma>1$ such that

$$
\liminf _{|\zeta| \rightarrow \infty} \frac{\Phi_{k}(\zeta)}{|\zeta|^{\gamma}}>0
$$

holds uniformly for $k$. Let $\xi, \xi_{k} \in \mathbb{R}$ be such that

$$
\lim _{k \rightarrow \infty}\left(\nabla \Phi_{k}\left(\xi_{k}\right)-\nabla \Phi(\xi)\right) \cdot\left(\xi_{k}-\xi\right)=0 .
$$

Then $\left\{\nabla \Phi_{k}\left(\xi_{k}\right)\right\}$ converges to $\nabla \Phi(\xi)$.

Proof. First, we prove that $\left\{\xi_{k}\right\}$ is bounded in $\mathbb{R}^{N}$. Note that the convexity of $\Phi_{k}$ implies

$$
\begin{aligned}
\Phi_{k}\left(\xi_{k}\right) & \leq \Phi_{k}(\xi)+\nabla \Phi_{k}\left(\xi_{k}\right) \cdot\left(\xi_{k}-\xi\right) \\
& =\Phi_{k}(\xi)+\nabla \Phi(\xi) \cdot\left(\xi_{k}-\xi\right)+\left(\nabla \Phi_{k}\left(\xi_{k}\right)-\nabla \Phi(\xi)\right) \cdot\left(\xi_{k}-\xi\right) .
\end{aligned}
$$

If $\left\{\xi_{k}\right\}$ were unbounded, using (7.2) and the fact that $\lim _{k \rightarrow \infty} \Phi_{k}(\xi)=\Phi(\xi)$, we would have

$$
\limsup _{k \rightarrow \infty} \frac{\Phi_{k}\left(\xi_{k}\right)}{\left|\xi_{k}\right|^{\gamma}} \leq 0
$$

which contradicts (7.1).

Now we use an argument borrowed from the proof of [22, Lemma 2.1]. Define $\hat{\Phi}_{k}(\zeta)=\Phi_{k}(\zeta)-\nabla \Phi_{k}(\xi) \cdot \zeta$ and $\hat{\Phi}(\zeta)=\Phi(\zeta)-\nabla \Phi(\xi) \cdot \zeta$ for $\zeta \in \mathbb{R}$. Then, since $\left\{\xi_{k}\right\}$ is bounded, $\hat{\Phi}_{k}$ and $\hat{\Phi}$ satisfy all the assumptions of the present lemma. Moreover, $\xi$ is a minimizer of both $\hat{\Phi}_{k}$ and $\hat{\Phi}$. Therefore, we may assume, without loss of generality, that $\xi$ is a minimizer of both $\Phi_{k}$ and $\Phi$, and we need to prove that $\lim _{k \rightarrow \infty}$ $\nabla \Phi_{k}\left(\xi_{k}\right)=0$.

Assume by contradiction that there exists $\delta>0$ such that, up to a subsequence, $\left|\nabla \Phi_{k}\left(\xi_{k}\right)\right|>\delta$ for every $k \in \mathbb{N}$. Let $t_{k} \in(0,1)$ be such that $\left|\nabla \Phi_{k}\left(\left(1-t_{k}\right) \xi+t_{k} \xi_{k}\right)\right|=\delta$ and let $\zeta_{k}=\left(1-t_{k}\right) \xi+t_{k} \xi_{k}$. Up to a subsequence, $\nabla \Phi_{k}\left(\zeta_{k}\right)$ is convergent to some $\alpha \in \mathbb{R}^{N}$ with $|\alpha|=\delta$. Since

$$
0 \leq\left(\nabla \Phi_{k}\left(\xi_{k}\right)-\nabla \Phi_{k}\left(\zeta_{k}\right)\right) \cdot\left(\xi_{k}-\zeta_{k}\right)=\left(1-t_{k}\right)\left(\nabla \Phi_{k}\left(\xi_{k}\right)-\nabla \Phi_{k}\left(\zeta_{k}\right)\right) \cdot\left(\xi_{k}-\xi\right),
$$

one has

$$
0 \leq \nabla \Phi_{k}\left(\zeta_{k}\right) \cdot\left(\zeta_{k}-\xi\right)=t_{k} \nabla \Phi_{k}\left(\zeta_{k}\right) \cdot\left(\xi_{k}-\xi\right) \leq t_{k} \nabla \Phi_{k}\left(\xi_{k}\right) \cdot\left(\xi_{k}-\xi\right) .
$$

This, together with (7.2), implies

$$
\lim _{k \rightarrow \infty} \nabla \Phi_{k}\left(\zeta_{k}\right) \cdot\left(\zeta_{k}-\xi\right)=0 .
$$

Taking the limit in the inequality

$$
\Phi_{k}(\xi) \geq \Phi_{k}\left(\zeta_{k}\right)+\nabla \Phi_{k}\left(\zeta_{k}\right) \cdot\left(\xi-\zeta_{k}\right)
$$

and using (7.3), since $\lim _{k \rightarrow \infty} \Phi_{k}(\xi)=\Phi(\xi)$ and since $\xi$ is a minimizer of $\Phi_{k}$, we see that

$$
\lim _{k \rightarrow \infty} \Phi_{k}\left(\zeta_{k}\right)=\Phi(\xi)
$$

Copyright $@$ by SIAM. Unauthorized reproduction of this article is prohibited. 
For every $\eta \in \mathbb{R}^{N}$, passing to the limit as $k \rightarrow \infty$ in the inequality

$\Phi_{k}(\eta) \geq \Phi_{k}\left(\zeta_{k}\right)+\nabla \Phi_{k}\left(\zeta_{k}\right) \cdot\left(\eta-\zeta_{k}\right)=\Phi_{k}\left(\zeta_{k}\right)+\nabla \Phi_{k}\left(\zeta_{k}\right) \cdot(\eta-\xi)+\nabla \Phi_{k}\left(\zeta_{k}\right) \cdot\left(\xi-\zeta_{k}\right)$

and using (7.3), (7.4), and the fact that $\lim _{k \rightarrow \infty} \nabla \Phi_{k}\left(\zeta_{k}\right)=\alpha$, we arrive at

$$
\Phi(\eta) \geq \Phi(\xi)+\alpha \cdot(\eta-\xi) \quad \forall \eta \in \mathbb{R}^{N} .
$$

Since $\alpha \neq 0$, this contradicts the fact that $\xi$ is a minimizer of $\Phi$ and $\Phi$ is of class $C^{1}$.

Acknowledgments. The authors would like to thank the anonymous referees for their stimulating comments and suggestions.

\section{REFERENCES}

[1] R. Adams and J. F. Fournier, Sobolev Spaces, 2nd ed., Pure and Applied Mathematics 140, Elsevier/Academic Press, Amsterdam, 2003.

[2] L. Boccardo And F. Murat, Almost everywhere convergence of the gradients of solutions to elliptic and parabolic equations, Nonlinear Anal., 19 (1992), pp. 581-597.

[3] L. Brasco, G. Carlier, and F. Santambrogio, Congested traffic dynamics, weak flows and very degenerate elliptic equations, J. Math. Pures Appl., 93 (2010), pp. 652-671.

[4] A. Canino and M. Degiovanni, Nonsmooth critical point theory and quasilinear elliptic equations, in Topological Methods in Differential Equations and Inclusions, NATO Science Ser. C 472, Springer, Dordrecht, 1995, pp. 1-50.

[5] K.-C. Chang, Infinite Dimensional Morse Theory and Multiple Solution Problems, Progress in Nonlinear Differential Equations and their Applications 6, Birkhäuser, Boston, MA, 1993.

[6] D. C. Clark, A variant of the Lusternik-Schnirelman theory, Indiana Univ. Math. J., 22 (1973), pp. 65-74.

[7] C. V. Coffman, A minimum-maximum principle for a class of non-linear integral equations, J. Analyse Math., 22 (1969), pp. 391-419.

[8] M. Colombo And A. Figalli, Regularity results for very degenerate elliptic equations, J. Math. Pures Appl., 101 (2014), pp. 94-117.

[9] J.-N. Corvellec, M. Degiovanni, and M. Marzocchi, Deformation properties for continuous functionals and critical point theory, Topol. Methods Nonlinear Anal., 1 (1993), pp. $151-171$.

[10] G. Dal Maso and F. Murat, Almost everywhere convergence of gradients of solutions to nonlinear elliptic systems, Nonlinear Anal., 31 (1998), pp. 405-412.

[11] M. Degiovanni, Variational methods for functionals with lack of strict convexity, in Nonlinear Equations: Methods, Models and Applications, Progress in Nonlinear Differential Equations and their Applications 54, D. Lupo, C. Pagani, and B. Ruf, eds., Birkhäuser, Boston, MA, 2003, pp. 127-130.

[12] M. Degiovanni, Critical groups of finite type for functionals defined on Banach spaces, Commun. Appl. Anal., 13 (2009), pp. 395-410.

[13] M. Degiovanni And M. Marzocchi, A critical point theory for nonsmooth functionals, Ann. Math. Pura Appl., 167 (1994), pp. 73-100.

[14] M. Degiovanni, M. Marzocchi, and V. D. Radulescu, Multiple solutions of hemivariational inequalities with area-type term, Calc. Var. Partial Differential Equations, 10 (2000), pp. $355-387$.

[15] M. Degiovanni And F. Schuricht, Buckling of nonlinearly elastic rods in the presence of obstacles treated by nonsmooth critical point theory, Math. Ann., 311 (1998), pp. 675-728.

[16] H. P. HeInz, Free Ljusternik-Schnirelman theory and the bifurcation diagrams of certain singular nonlinear problems, J. Differential Equations, 66 (1987) pp. 263-300.

[17] M. IZYdorek AND J. JancZeWsKa, Homoclinic solutions for a class of the second order Hamiltonian systems, J. Differential Equations, 219 (2005), pp. 375-389.

[18] R. KAJIKIYA, A critical point theorem related to the symmetric mountain pass lemma and its applications to elliptic equations, J. Funct. Anal., 225 (2005), pp. 352-370.

[19] J. LiU And Y. Guo, Critical point theory for nonsmooth functionals, Nonlinear Anal., 66 (2007), pp. 2731-2741.

[20] Z. LiU AND Z.-Q. WANG, Schrödinger equations with concave and convex nonlinearities, Z. Angew. Math. Phys., 56 (2005), pp. 609-629.

Copyright $@$ by SIAM. Unauthorized reproduction of this article is prohibited. 
[21] Z. LiU AND Z.-Q. WANG, On Clark's theorem and its applications to partially sublinear problems, Ann. Inst. H. Poincaré Anal. Non Linéaire, 32 (2015), pp. 1015-1037.

[22] P. MAGRone, An existence result for a problem with critical growth and lack of strict convexity, NoDEA Nonlinear Differential Equations Appl., 15 (2008), pp. 717-728.

[23] P. H. Rabinowitz, Minimax Methods in Critical Point Theory with Applications to Differential Equations, CBMS Regional Conference Series in Mathematics 65. American Mathematical Society, Providence, RI, 1986.

[24] P. H. Rabinowitz, Homoclinic orbits for a class of Hamiltonian systems, Proc. Roy. Soc. Edinburgh Sect. A., 114 (1990), pp. 33-38.

[25] M. Struwe, Variational Methods, Springer-Verlag, Berlin, 1996.

[26] Z.-Q. WANG, Nonlinear boundary value problems with concave nonlinearities near the origin, NoDEA Nonlinear Differential Equations Appl., 8 (2001), pp. 15-33.

[27] Z. Zhang, T. XIANG, AND R. YuAn, Homoclinic solutions for subquadratic Hamiltonian systems without coercive conditions, Taiwanese J. Math., 18 (2014), pp. 1089-1105.

Copyright (c) by SIAM. Unauthorized reproduction of this article is prohibited. 